IUCrJ

ISSN 2052-2525

BIOLOGY|MEDICINE

Received 5 May 2020

Accepted 15 July 2020

Edited by Z.-J. Liu, Chinese Academy of Sciences, China

Keywords: two-component regulatory systems; sensor histidine kinase; histidine-containing phosphotransfer proteins; Pseudomonas aeruginosa.

PDB references: PA1611REC-Mg ${ }^{2+}, 7 \mathrm{c} 1 \mathrm{j}$; PA1611REC-Ca ${ }^{2+}, 7 c f w ;$ WT-HptB, 7c1i

Supporting information: this article has supporting information at www.iucrj.org

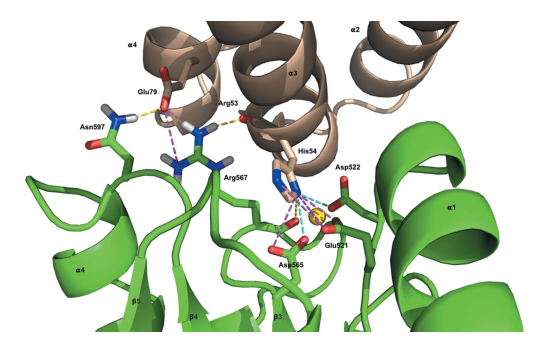

OPEN $\odot$ ACCESS

\section{Structural insights into the histidine-containing phosphotransfer protein and receiver domain of sensor histidine kinase suggest a complex model in the two-component regulatory system in Pseudomonas aeruginosa}

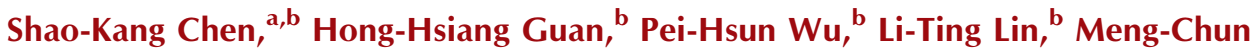 \\ Wu, ${ }^{\mathrm{a}, \mathrm{b}}$ Hwan-You Chang, ${ }^{\mathrm{c}}$ Nai-Chi Chen, ${ }^{\mathrm{b}}$ Chien-Chih Lin,, ${ }^{\mathrm{b}}$ Phimonphan \\ Chuankhayan, ${ }^{b}$ Yen-Chieh Huang, ${ }^{b}$ Pei-Ju Lin ${ }^{b, d}$ and Chun-Jung Chen ${ }^{a, b, e, f_{*}}$ \\ aDepartment of Biotechnology and Bioindustry Sciences, National Cheng Kung University, Tainan City 701, Taiwan, ${ }^{\mathbf{b}}$ Life
Science Group, Scientific Research Division, National Synchrotron Radiation Research Center, Hsinchu 30076, Taiwan,
'Institute of Molecular Medicine, National Tsing Hua University, Hsinchu 30013, Taiwan, ${ }^{\mathbf{d}}$ Institute of Bioinformatics and
Structural Biology, National Tsing Hua University, Hsinchu 30013, Taiwan, ${ }^{\mathbf{e} D e p a r t m e n t ~ o f ~ P h y s i c s, ~ N a t i o n a l ~ T s i n g ~ H u a ~}$
University, Hsinchu 30013, Taiwan, and ${ }^{\mathbf{f} D e p a r t m e n t ~ o f ~ B i o l o g i c a l ~ S c i e n c e ~ a n d ~ T e c h n o l o g y, ~ N a t i o n a l ~ C h i a o ~ T u n g ~}$
University, Hsinchu 30010, Taiwan. ${ }^{*}$ Correspondence e-mail: cjchen@nsrrc.org.tw
}

In Pseudomonas aeruginosa, an important opportunistic pathogen that causes numerous acute and chronic infections, the hybrid two-component system (TCS) regulates the swarming ability and biofilm formation with a multistep phosphorelay, and consists of hybrid-sensor histidine kinase (HK), histidinecontaining phosphotransfer protein (Hpt) and response regulator (RR). In this work, two crystal structures of HptB and the receiver domain of HK PA1611 (PA1611REC) of $P$. aeruginosa have been determined in order to elucidate their interactions for the transfer of the phosphoryl group. The structure of HptB folds into an elongated four-helix bundle - helices $\alpha 2, \alpha 3, \alpha 4$ and $\alpha 5$, covered by the short $\mathrm{N}$-terminal helix $\alpha 1$. The imidazole side chain of the conserved activesite histidine residue His57, located near the middle of helix $\alpha 3$, protrudes from the bundle and is exposed to solvent. The structure of PA1611REC possesses a conventional $(\beta / \alpha)_{5}$ topology with five-stranded parallel $\beta$-sheets folded in the central region, surrounded by five $\alpha$-helices. The divalent $\mathrm{Mg}^{2+}$ ion is located in the negatively charged active-site cleft and interacts with Asp522, Asp565 and Arg567. The HptB-PA1611REC complex is further modeled to analyze the binding surface and interactions between the two proteins. The model shows a shape complementarity between the convex surface of PA1611REC and the kidney-shaped $\mathrm{HptB}$ with fewer residues and a different network involved in interactions compared with other TCS complexes, such as SLN1-R1/YPD1 from Saccharomyces cerevisiae and AHK $5_{\mathrm{RD}} / \mathrm{AHP} 1$ from Arabidopsis thaliana. These structural results provide a better understanding of the TCS in $P$. aeruginosa and could potentially lead to the discovery of a new treatment for infection.

\section{Introduction}

In aiding the adaptation of cells to environmental changes, two-component regulatory systems (TCS) are widely distributed in prokaryotes, whereas few are identified in lower eukaryotic organisms and plants. The basic mechanism of the TCS signaling transduction is the transfer of a phosphoryl group, which serves as a signaling molecule. The system is composed of a sensor histidine kinase (HK), which is autophosphorylated at a conserved histidine residue of its cytoplasmic transmitter domain after sensing outside signals with the extracellular input domain; and a response regulator 
(RR), which accepts the phosphoryl group at the conserved aspartate residue of the receiver domain (REC) from HK or a histidine-containing phosphotransfer protein $(\mathrm{Hpt})$ (Stock et al., 1989; 2000). The RR protein initiates the response by binding to a specific target protein, such as a transcriptional factor or DNA element (Bell et al., 2010; Bauer et al., 2013). The chemistry of the phosphoramide and acylphosphate linkage is involved in this mechanism (Thomason \& Kay, 2000). Biofilm formation and the implementation of drug resistance are typically regulated by TCS, which makes TCS an attractive target for research and drug intervention (Anjali et al., 2019).

The sensor-regulator protein pairs in a TCS can be classified into three major types based on the organization of functional domains of HK: classic, unorthodox and hybrid systems (Rodrigue et al., 2000; Lin et al., 2006). In the classic system, a phosphate is transferred directly from phosphorylated HK to RR (Skerker et al., 2008; Capra et al., 2010). In the unorthodox system, an extra receiver domain (REC) and a histidine-containing phosphotransfer (Hpt) domain are sequentially connected to the C-terminus of the HK transmitter domain. After the extracellular input domain of HK senses signals from the environment, the transmitter domain autophosphorylates; the phosphate is then relayed to the receiver domain and the Hpt domain of HK. Subsequently, the Hpt domain transphosphorylates the conserved aspartate residue at the receiver domain of RR (Posas et al., 1996; Thomason \& Kay, 2000; Zhao et al., 2008; Kaserer et al., 2010). The hybrid system is similar to the unorthodox system, but, instead of an Hpt domain attached to HK, an independent Hpt protein transfers the phosphoryl group to the downstream RR (Rodrigue et al., 2000).

Pseudomonas aeruginosa ( $P$. aeruginosa) is a versatile Gram-negative bacterium grown in soil, coastal marine habitats, and on plant and animal tissues (Stover et al., 2000). It is an elongated rod-shaped bacterium with only one-way flagellum mobility. Previous studies have shown that the metabolic versatility, intrinsic and acquired antibiotic resistance, biofilm formation and production of multiple virulence factors make $P$. aeruginosa a formidable pathogen causing numerous acute and chronic infections (Balasubramanian et al., 2013). It accounts for $10-20 \%$ of all hospital-acquired infections and is the leading cause of chronic pulmonary infections and mortality in cystic fibrosis (CF) patients and burn victims (Stover et al., 2000; National Nosocomial Infections Surveillance System, 2004; Lyczak et al., 2002). In $P$. aeruginosa, the Hpt-mediated hybrid TCS serves as a basic stimulus-response coupling mechanism to decrease the environmental harm and to ensure survival by activating downstream responses, including antibiotic susceptibility, swarming activity and biofilm formation (Stock et al., 2000; Wolanin et al., 2002). Many TCS components have been described for their key roles during the infection processes of P. aeruginosa (Rodrigue et al., 2000).

The three orphan Hpt proteins in P. aeruginosa are HptA, $\mathrm{HptB}$ and HptC. HptB, the target protein in this work, is closely related to biofilm formation, which could increase drug resistance and protect the cell from pinocytosis by the host. According to previous studies, after the sensor proteins PA1611, PA1976 and PA2824 are autophosphorylated, the phosphoryl group is transferred to $\mathrm{HptB}$ and subsequently to the downstream RR protein PA3346 as illustrated in Fig. 1. The results showed that the HptB-mediated multistep phosphorelay plays a central role in the infection process of P. aeruginosa (Lin et al., 2006; Hsu et al., 2008). Moreover, the previous structures of $\mathrm{Hpt}$ proteins/domains in complex with receiver domains of HK or RR, such as SLN1-R1/YPD1 of yeast (PDB entry 2r25) and AHK5 $5_{\mathrm{RD}} / \mathrm{AHP} 1$ of Arabidopsis thaliana (PDB entry 4euk; Bauer et al., 2013), have shown that the Hpt protein/domain would interact with the receiver domain. To elucidate the HptB-mediated TCS pathway of $P$. aeruginosa in depth, we report two crystal structures of HptB and the C-terminal receiver domain of HK PA1611 (PA1611REC). The structural details allow us to model the complex of HptB and PA1611REC, which reveals the possible binding conformation and the residues involved in the interaction between the two proteins. The result would further clarify the mechanism in the HptB-mediated signal

\section{Sensor histidine kinase}

(PA1611, PA1976, PA2824, PA4856)

HptB

Response Regulator (PA3346)

\section{Antisigma} antagonist (PA3347)

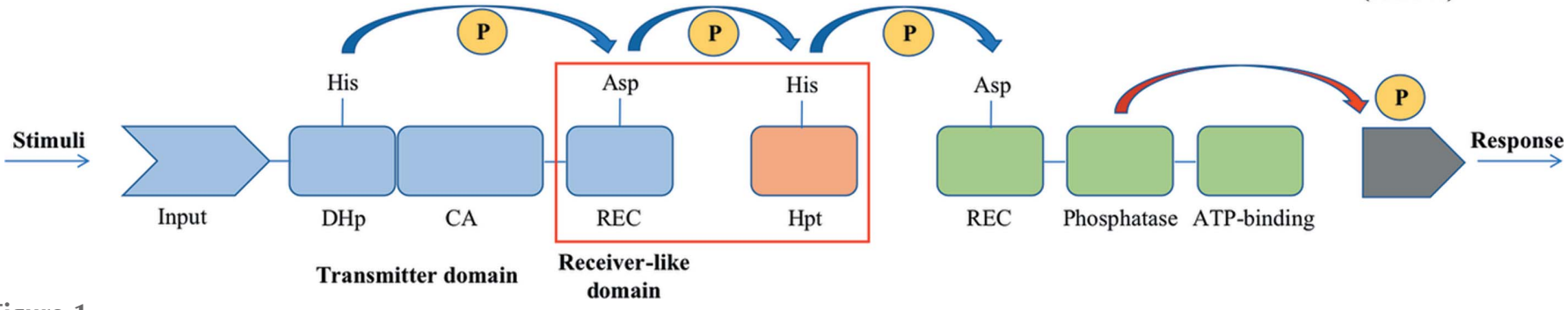

Figure 1

Schematic diagram of the phosphorelay in HptB-mediated hybrid TCS. The P. aeruginosa gene index numbers of the orphan sensors and the response regulator are listed in parentheses. Blue arrows indicate the phosphoryl transfer in the pathway. The phosphoryl group is presented with an encircled P. After activation with environmental stimuli, sensor histidine kinases (PA1611, PA1976, PA2824 and PA4856) autophosphorylate and transfer the phosphoryl group specifically to HptB, which in turn relays the signal to response regulator PA3346. The phosphorylation regulates the Ser/Thr phosphatase activity of PA3346, resulting in increased phosphatase activity and dephosphorylation of the anti-sigma antagonist PA3347 (indicated with a red arrow). The phosphorylation/dephosphorylation status might modulate the binding activity of PA3347 to other factor(s) and lead to the regulation of genes associated with swarming activity and biofilm formation. 
Table 1

The DNA primers used in this study.

\begin{tabular}{lll}
\hline Primers & Sequence $\left(5^{\prime} \rightarrow 3^{\prime}\right)$ & Comment \\
\hline HptB-C75A-Fw & CTCCTCGCCGGCTACGCGAAGGAGCTGGAGGAAAG & Mutation of HptB Cys75 to Ala \\
HptB-C75A-Rv & CTTTCCTCCAGCTCCTTCGCGTAGCCGGCGAGGAG & Mutation of HptB Cys75 to Ala \\
PA1611REC-Fw & CTAGCTAGCGCGCCGCCCG & Cloning of PA1611REC into pET28a \\
PA1611REC-Rv & GGAATTCTCATTCCGGCTCCCC & Cloning of PA1611REC into pET28a \\
\hline
\end{tabular}

transduction pathway of $P$. aeruginosa and potentially lead to the discovery of a new treatment for $P$. aeruginosa infection, such as novel small molecules which could potentially interfere with the interaction between $\mathrm{HptB}$ and the corresponding receiver domains.

\section{Materials and methods}

\subsection{Cloning and expression of target proteins}

Native HptB was cloned into pET23a (Novagen) using $\mathrm{NdeI}$ and XhoI restriction sites. For the mutant HptB, the only cysteine (C75) was mutated to alanine by polymerase chain reaction (PCR) overlap extension. The $\mathrm{C}$-terminal fragment (a.a. 507-651) including the receiver domain of HK PA1611 (PA1611REC) was amplified by PCR and cloned into pET28a (Novagen) using NheI and EcoRI restriction sites with T4 DNA ligase (Invitrogen). The primers used are listed in Table 1. E. coli strain DH5 $\alpha$ was used for the plasmid preparation and recombinant DNA manipulation.

After DNA sequencing confirmed the correctness of the insert, the expression constructs of both native HptB and PA1611REC were transformed into E. coli strain BL21(DE3) for the expression of recombinant proteins. The bacteria were cultured with shaking in Luria-Bertani media supplemented with specific antibiotics $\left(100 \mu \mathrm{g} \mathrm{ml}^{-1}\right)$ until the optical density at $600 \mathrm{~nm}$ (OD600) reached 0.6. Overexpression of the target protein was induced on adding isopropyl- $\beta$-D-thiogalactoside (IPTG) to the final concentrations of $0.5 \mathrm{~m} M$ and $0.1 \mathrm{~m} M$ for native HptB and PA1611REC, respectively. The culture was grown overnight at $20^{\circ} \mathrm{C}$ with shaking and harvested using centrifugation at $8000 \mathrm{~g}$ for $25 \mathrm{~min}$ at $4^{\circ} \mathrm{C}$.

For the production of selenomethionine-labeled (SeMet) mutant HptB, E. coli strain BL21-Gold (DE3) harboring the expression vector served as the host cell. The culture was grown in M9 medium $\left(6 \mathrm{mg} \mathrm{l}^{-1} \mathrm{Na}_{2} \mathrm{HPO}_{4}, 3 \mathrm{mg} \mathrm{l}^{-1} \mathrm{KH}_{2} \mathrm{PO}_{4}\right.$, $0.5 \mathrm{mg} \mathrm{l}^{-1} \mathrm{NaCl}, 1 \mathrm{mg} \mathrm{l}^{-1} \mathrm{NH}_{4} \mathrm{Cl}, 1 \mathrm{~m} M \mathrm{MgSO}_{4}, 0.1 \mathrm{~m} M$ $\mathrm{CaCl}_{2}, 1 \mathrm{mg} \mathrm{l}^{-1}$ thiamine- $\mathrm{HCl}, 0.2 \%$ glucose) with L-lysine, Lthreonine, L-phenylalanine (120 $\left.\mathrm{mg} \mathrm{l}^{-1}\right)$, L-leucine, L-isoleucine, L-valine, L-selenomethionine $\left(60 \mathrm{mg} \mathrm{l}^{-1}\right)$ and ampicillin $\left(100 \mu \mathrm{g} \mathrm{ml}^{-1}\right)$ until the optical density at $600 \mathrm{~nm}$ (OD600) reached 0.6. The following steps for the expression and purification of the SeMet-labeled protein were the same as the native HptB protein.

\subsection{Purification of $H p t B$ protein}

The cell pellet from a 11 culture was suspended in a lysis buffer $(35 \mathrm{ml})$ consisting of Tris- $\mathrm{HCl}(50 \mathrm{mM}, \mathrm{pH} 8.0)$ and subjected to cell disruption by ultrasonication using a pulsation cycle $2 \mathrm{~s}$ on and $3 \mathrm{~s}$ off with a total duration of $20 \mathrm{~min}$ at $40 \%$ energy on ice. The soluble protein extract was collected by centrifugation at $12000 \mathrm{~g}$ for $30 \mathrm{~min}$ at $4{ }^{\circ} \mathrm{C}$ and passed through an open column packed with resin (Q Sepharose Fast Flow, $60 \mathrm{ml}$, GE Healthcare Bio-Sciences AB, Uppsala, Sweden), which was equilibrated with binding buffer (90 ml) consisting of Tris- $\mathrm{HCl}(50 \mathrm{mM}, \mathrm{pH} 8.0)$. The column was washed with binding buffer $(90 \mathrm{ml})$ containing $\left(\mathrm{NH}_{4}\right)_{2} \mathrm{SO}_{4}$ $(40 \mathrm{mM})$. The target protein was eluted with portions $(90 \mathrm{ml})$ of binding buffer containing $\left(\mathrm{NH}_{4}\right)_{2} \mathrm{SO}_{4}$ at stepwise-increasing concentrations of 60 and $100 \mathrm{mM}$. The eluted fractions were concentrated by the centricon of a $3 \mathrm{kDa}$ filter unit (Amicon Ultra, Merck KGaA, Darmstadt, Germany) and then loaded onto a column (HiLoad 16/60 Superdex 75 PG, GE Healthcare Bio-Sciences $\mathrm{AB}$ ) connected to a liquid chromatography (FPLC) system (ÄKTA, GE Healthcare) and equilibrated with a buffer composed of $\mathrm{NaCl}(300 \mathrm{mM})$ and Tris- $\mathrm{HCl}$ (20 $\mathrm{m} M, \mathrm{pH}$ 8.0). The size-exclusion chromatography step yielded homogenous protein fractions.

\subsection{Purification of PA1611REC protein}

The procedures of cell disruption and collection of the soluble protein extract were the same as those for the purification of $\mathrm{HptB}$ except for the lysis buffer, which was composed of $\mathrm{NaCl}(500 \mathrm{~m} M)$ and Tris- $\mathrm{HCl}(50 \mathrm{~m} M, \mathrm{pH} 8.0)$. The extract was then passed through an open column packed with resin (10 ml Ni-Sepharose 6 Fast Flow, GE Healthcare Bio-Sciences AB, Uppsala, Sweden), which was equilibrated with a binding buffer $(50 \mathrm{ml})$ consisting of $\mathrm{NaCl}(500 \mathrm{mM})$ and Tris- $\mathrm{HCl}(50 \mathrm{~m} M, \mathrm{pH} 8.0)$. The column was washed with a binding buffer $(50 \mathrm{ml})$ containing imidazole $(20 \mathrm{mM})$. The target protein was eluted with portions $(50 \mathrm{ml})$ of binding buffer containing imidazole at stepwise-increasing concentrations of $80,100,300$ and $500 \mathrm{~m} M$.

\subsection{Crystallization}

After the eluted fractions were collected and verified with SDS-PAGE (15\%) followed by staining (Coomassie Brilliant Blue R-250), the purified $\mathrm{HptB}$ protein was dialyzed against Tris- $\mathrm{HCl}\left(50 \mathrm{mM}, \mathrm{pH} \mathrm{7.5)}\right.$ and concentrated to $\sim 8 \mathrm{mg} \mathrm{ml}^{-1}$ with a Centricon $3 \mathrm{kDa}$ filter unit (Amicon Ultra, Merck KGaA, Darmstadt, Germany), whereas the purified PA1611REC protein was dialyzed against distilled water and concentrated to $\sim 10 \mathrm{mg} \mathrm{ml}^{-1}$ by the same method. Initial screening of crystallization conditions was performed by the hanging-drop vapor-diffusion method in 96-well plates with a liquid-handling robot (Mosquito, TTP Labtech). The hanging 
Table 2

Data collection and refinement statistics.

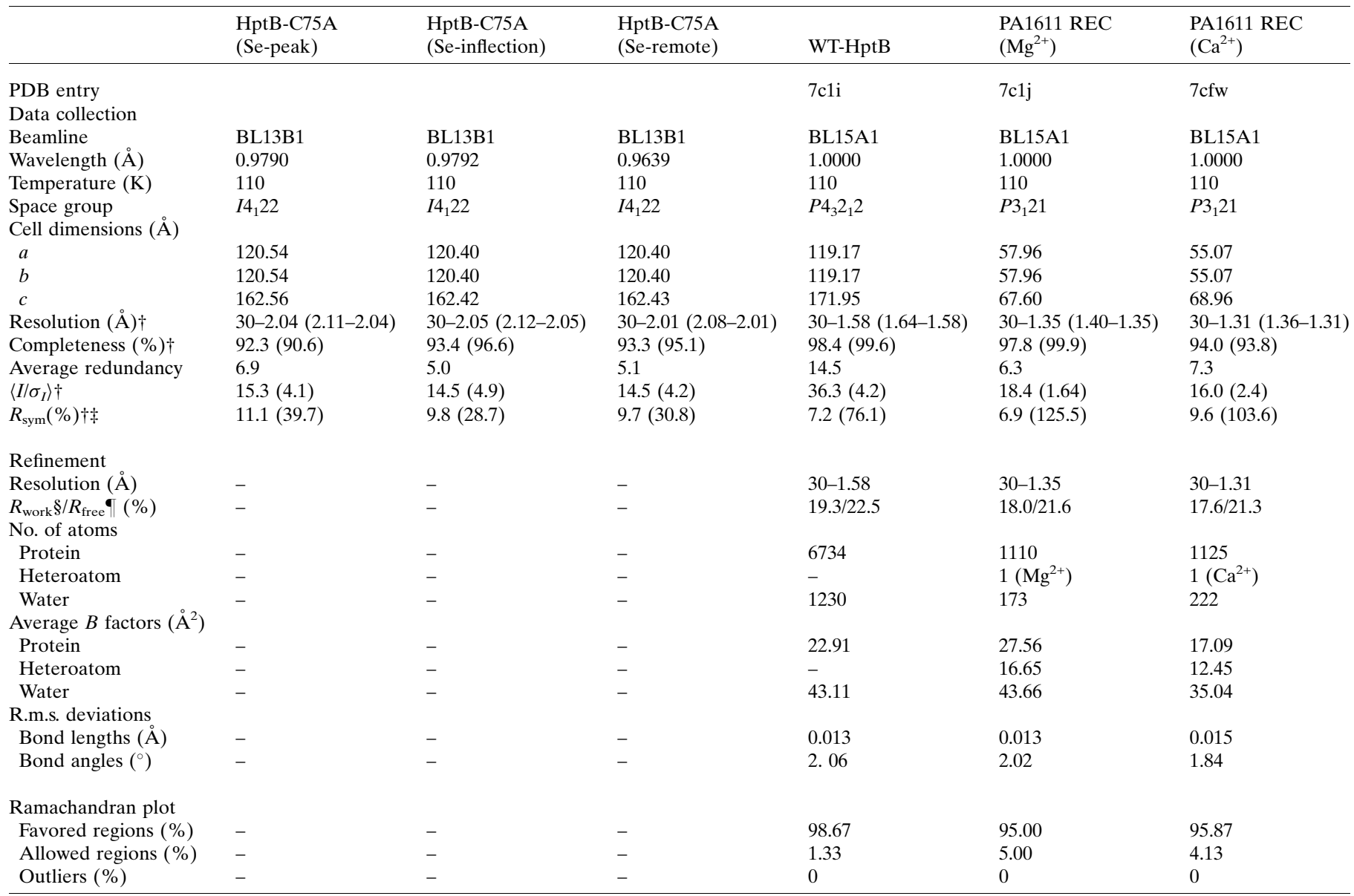

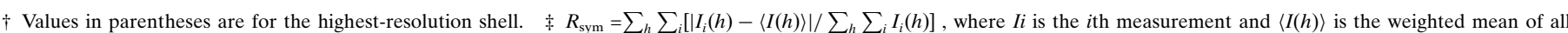

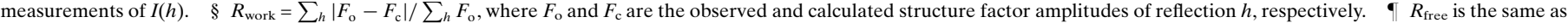
$R_{\text {work }}$, but calculated with $5 \%$ of randomly chosen reflections omitted from refinement.

drops formed on mixing protein solution $(0.1 \mu \mathrm{l})$ with reservoir solution $(0.1 \mu \mathrm{l})$ and equilibrated against the reservoir solution $(100 \mu \mathrm{l})$. To optimize the protein crystals, the hanging drops were made on mixing a protein solution $(1 \mu \mathrm{l})$ with a reservoir solution $(1 \mu \mathrm{l})$ and equilibrated against a reservoir solution $(120 \mu \mathrm{l})$ in VDX48 plates (Hampton Research). All plates were placed in an incubator at $18^{\circ} \mathrm{C}$.

Crystals formed under several conditions. The crystal of SeMet-labeled mutant $\mathrm{HptB}$, grown in a reservoir solution containing PEG20000 $[12 \%(w / v)]$ and MES monohydrate (0.1 $M, \mathrm{pH}$ 6.5), was picked for data collection. The structure of native $\mathrm{HptB}$ determined was from the crystals grown in a reservoir solution containing PEG6000 $[10 \%(w / v)]$ and bicine/ sodium hydroxide $(100 \mathrm{~m} M, \mathrm{pH} 9.0)$. For PA1611REC, the structure determined was from crystals grown in a reservoir solution containing PEG4000 $[15 \%(w / v)], \mathrm{MgCl}_{2}(0.2 M)$ and Tris- $\mathrm{HCl}(100 \mathrm{~m} M, \mathrm{pH} 8.5)$.

\subsection{Data collection and processing}

The crystals were transferred from the hanging drops into a cryoprotectant solution $(1 \mu \mathrm{l})$ made from adding glycerol
$[20 \%(v / v)]$ into the reservoir solution for a few seconds. The crystals were then picked with synthetic nylon loops (Hampton Research, Aliso Viejo, CA, USA) and flash-cooled in liquid nitrogen. The X-ray diffraction datasets were collected on beamlines TPS 05A, BL13B1 and BL15A1 at the National Synchrotron Radiation Research Center (NSRRC), Taiwan, and BL44XU and BL12B2 at SPring-8, Japan. For SeMet-labeled HptB, a multi-wavelength anomalousdispersion (Se-MAD) experiment was performed. All datasets were indexed, integrated and scaled using the HKL2000 program suite (Otwinowski \& Minor, 1997). The data collection statistics are listed in Table 2.

\subsection{Structure determination and refinement}

The positions of nine selenium atoms of three SeMetlabeled mutant HptB-C75A molecules in the asymmetric unit of space group $I 4_{1} 22$ were automatically determined and used to calculate the multi-wavelength anomalous-diffraction phases with SOLVE (Terwilliger \& Berendzen, 1999). The phases of wild-type $\mathrm{HptB}$ in space group $P 4_{3} 2_{1} 2$ and PA1611REC in space group $P 3_{1} 21$ were determined with the 
molecular replacement method using Phaser MR in the CCP4 program suite (Winn et al., 2011), with the structures of $a b$ inito determined mutant $\mathrm{HptB}-\mathrm{C} 75 \mathrm{~A}$ and the receiver domain from yeast histidine kinase SLN1 (PDB entry 2r25; B-chain) as the search models, respectively. Structural refinement was performed using alternating rounds of model building with the program Coot (Emsley et al., 2010) and restrained refinement with Refmac5 in the CCP4 program suite (Murshudov et al., 2011) to improve the value of the $R$ factor and $R_{\text {free }}$ The refinement statistics are listed in Table 2. The secondary structure was assigned with the DSSP web server (Kabsch \& Sander, 1983; Touw et al., 2015), and the structure figures were prepared using PyMOL (http://www.pymol.org/).

\subsection{Modeling of the HptB-PA1611REC complex}

To establish the complex model of HptB and PA1611REC, docking was performed using the crystal structures of two individual proteins with the $H A D D O C K$ web server (version 2.2; van Zundert et al., 2016) in the easy interface mode. His57 of HptB and Asp565 of PA1611REC were considered the active residues because these two residues directly participate in the transfer of the phosphoryl group according to previous studies, which confirmed this transfer between PA1611 and HptB (Lin et al., 2006; Hsu et al., 2008). The passive residues, which potentially make contact in the complex, were automatically defined in the easy interface mode that included all residues within a radius of $6.5 \AA$ from the active residue and on the surface with relative surface accessibility of either a main chain or a side chain above $15 \%$ as determined with NACCESS (Hubbard \& Thornton, 1993). The divalent metal ions $\left(\mathrm{Mg}^{2+}\right)$ in the active-site cleft of PA1611REC were also applied to the $H A D D O C K$ web server. The complex models were clustered according to the pairwise backbone root-meansquare deviation (RMSD) at the interface; the clusters were sorted according to a HADDOCK score calculated from energies of van der Waals, electrostatic interaction, desolvation, restraint violation and buried surface area. The HADDOCK web server clustered 115 structures into eight clusters, which represents $57.5 \%$ of the water-refined models generated by HADDOCK for the HptB-PA1611REC complex with $\mathrm{Mg}^{2+}$. The cluster with the lowest HADDOCK score is the most likely solution; the first model in this cluster was chosen for the analysis of the interface with the $P D B e$ PISA web server (Krissinel \& Henrick, 2007). The score of cluster 1 is $-63.7 \pm 2.6$, whereas other clusters range from -48 to -7.6 , indicating that the highest score is a unique solution.

\section{Results}

\subsection{Overall structure of $\mathrm{HptB}$}

HptB consists of 116 amino acids with a total molecular mass of $\sim 13.2 \mathrm{kDa}$ and shares a low-sequence identity with other Hpt proteins or domains [Fig. 2(a)]. Initially, we found crystallizing native $\mathrm{HptB}$ to be difficult. We suspected that Cys75 would introduce an intermolecular disulfide bond and interrupt the regular orientation of the protein in solution. We thus prepared SeMet-labeled mutant HptB-C75A for crystallization and phase determination. The crystals of SeMetlabeled mutant $\mathrm{HptB}-\mathrm{C} 75 \mathrm{~A}$ belong to the tetragonal space group $I 4_{1} 22$ with three protein molecules in one asymmetric unit. Then, we successfully crystallized the native HptB in
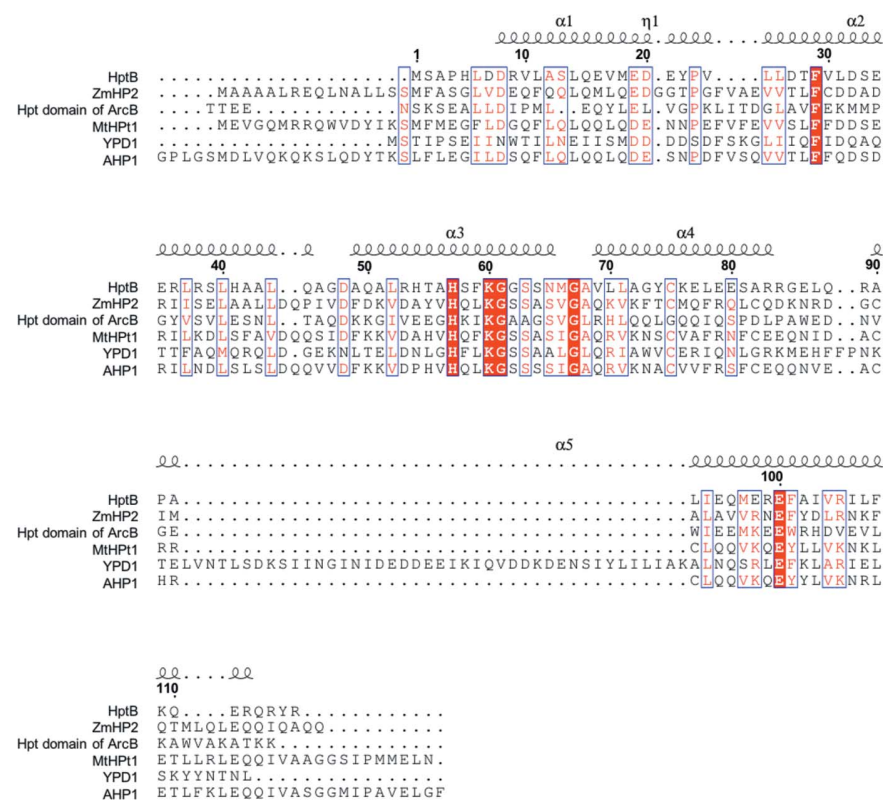

(a)
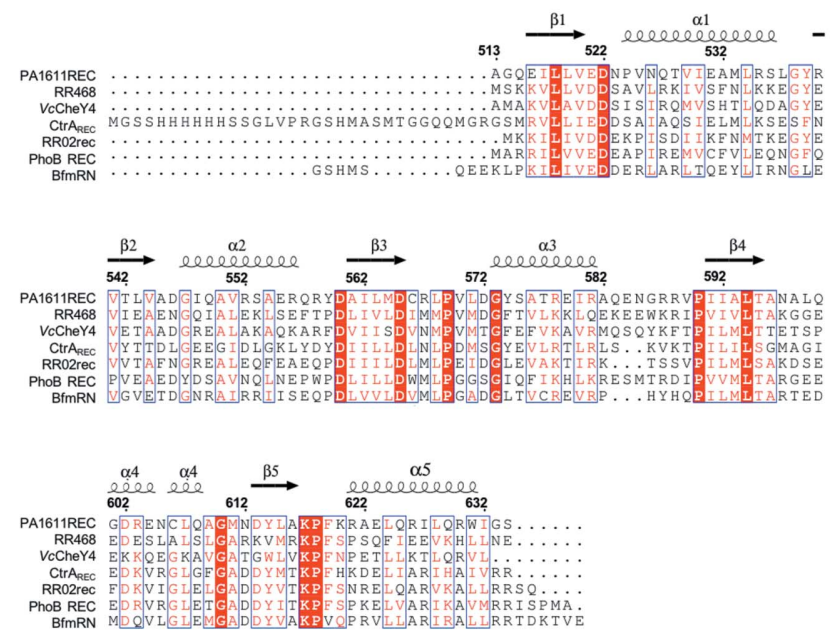

(b)

Figure 2

Information about secondary structures and sequence alignment of $(a)$ HptB and (b) PA1611REC with structural homologues obtained using the $D A L I$ server. The sequence comparison was performed with the ESPript web server (http://espript.ibcp.fr/ESPript/ESPript/). The residues with high similarity appear in blue boxes; the conserved residues are white shaded in red. The species and PDB codes of each protein sequence are as follows: ZmHP2, Zea mays, 1wn0 (Sugawara et al., 2005); Hpt domain of ArcB, Escherichia coli, 2a0b (Kato et al., 1999b); MtHPt1, Medicago truncatula, 3 us6 (Ruszkowski et al., 2013); YPD1, Saccharomyces cerevisiae, 2r25 (Zhao et al., 2008); AHP1, Arabidopsis thaliana, 4euk (Bauer et al., 2013); RR468, Thermotoga maritima, $3 \mathrm{gl9}$ (Casino et al., 2009); VcCheY4, Vibrio cholerae, $4 \mathrm{~h} 60$ (Biswas et al., 2013); $\mathrm{Ctr}_{\mathrm{REC}}$, Brucella abortus, 4qpj (Willet et al., 2015); RR02rec, Streptococcus pneumoniae, 1nxw (Bent et al., 2004); PhoB REC, Escherichia coli, $1 \mathrm{~b} 00$ (Sola et al., 1999); BfmRN, Acinetobacter baumannii, 6br7 (Draughn et al., 2018). 
another tetragonal space group, $P 4_{3} 22_{1} 2$, with six protein molecules in one asymmetric unit. The two pairs of trimeric proteins related to the non-crystallographic twofold symmetry exhibit similar structures [Fig. 3(a)], but the protein is monomeric in solution, as confirmed by size-exclusion chromatography. The initial phases and structure of SeMet-labeled mutant HptB-C75A were first determined with the Se-MAD method at $\sim 2 \AA$. The crystal structure of native HptB was subsequently determined with the mutant $\mathrm{HptB}$ structure as an initial model and refined to high resolution $(1.58 \AA)$. The structural differences between mutant and native HptB are small, with an RMSD of $0.15 \AA$. The structures of six native $\mathrm{HptBs}$ in one asymmetric unit are essentially the same, with a small RMSD of $0.16 \AA$ overall for the $\mathrm{C} \alpha$ atoms [Fig. 3(b)].
The final atomic model of native $\mathrm{HptB}$ comprises 115 residues, except for the first methionine. The entire main chain and most side chains are well defined with clear electron density.

The structure of HptB comprises five $\alpha$-helices and folds into an elongated $\alpha$-helical bundle with an up-and-down topology. The helices $\alpha 2$ (Tyr22-Gln45), $\alpha 3$ (Ala49-Asn65), $\alpha 4$ (Val69-Arg83) and $\alpha 5$ (Ala90-Arg112) are twisted around the central axis and form an antiparallel four-helix bundle. Residues Ala46-Asp48, Met66-Ala68 and Arg84-Arg89 form short turns between helices $\alpha 2-\alpha 3, \alpha 3-\alpha 4$ and $\alpha 4-\alpha 5$, respectively. The four-helix bundle is covered with the short $\mathrm{N}$-terminal helix $\alpha 1$ (Asp8-Met18) which protrudes into the solvent environment and connects to helix $\alpha 2$ with a $3_{10}$ helix (Glu19-Glu21) [Fig. 3(c)]. All these inter-helical regions are (a)

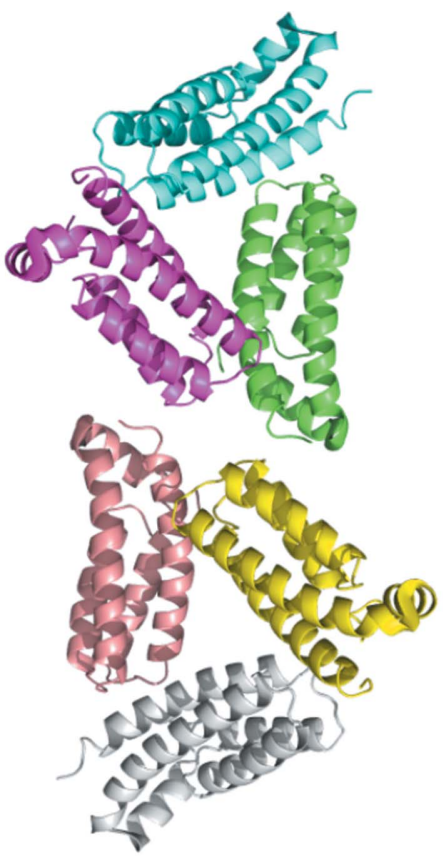

(d)

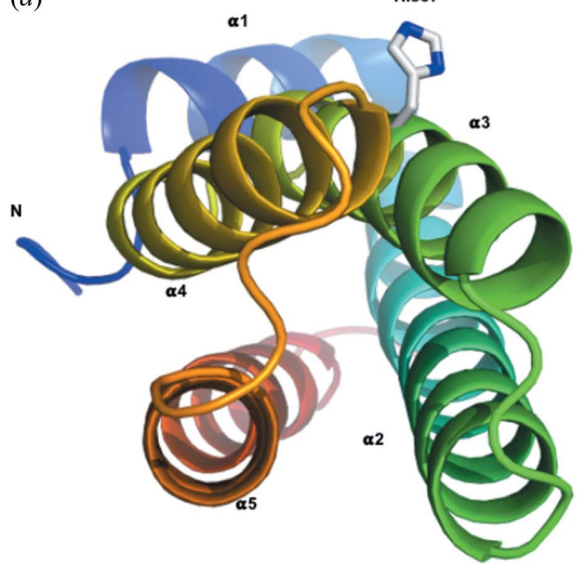

(b)

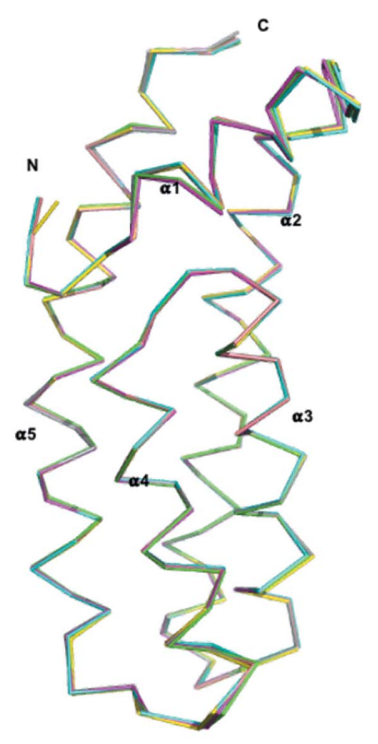

(c)

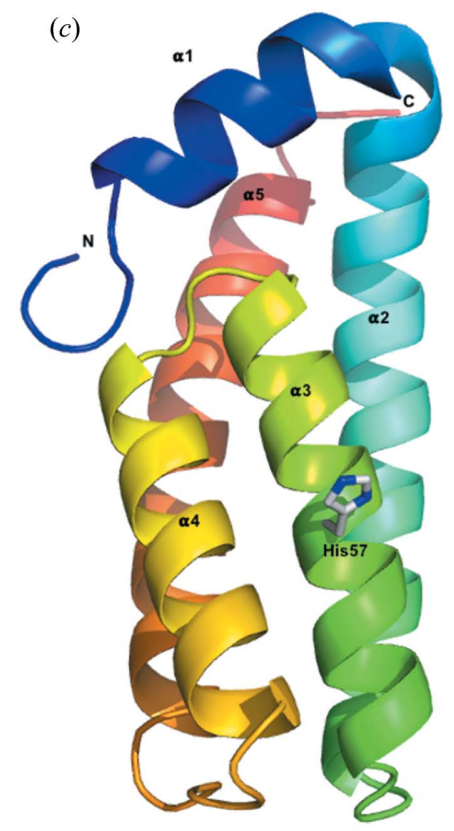

(e)

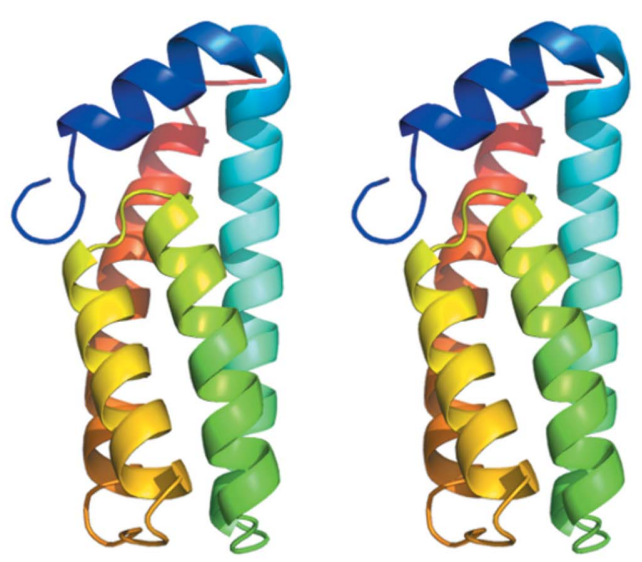

Figure 3

Overall structure of HptB. (a) Ribbon diagram illustrating the crystal packing in the native HptB crystal. There are six protein molecules in one asymmetric unit. (b) Diagram showing the structures are well superimposed among the six HptB protomers in the asymmetric unit. (c) Ribbon diagram illustrating the structural folding and secondary-structure elements of the HptB monomer. The helices are numbered sequentially $\alpha 1$ to $\alpha 5$ from the Nterminus to the C-terminus. The side-chain carbon and nitrogen atoms of the active-site histidine (His57) are shown as white and blue sticks, respectively. $(d)$ Bottom view of the structure from $(c)$. The side chain of His57 is highly exposed to solvent. The four-helix bundle core is composed of helices $\alpha 2, \alpha 3$, $\alpha 4$ and $\alpha 5$. (e) Stereoview of the HptB structure. Helix $\alpha 1$ forms a cap on top of the four-helix bundle. 
well ordered with some residues contributing to the stabilization of the helix-bundle through hydrogen bonds (Fig. 4).

The four-helix bundle is stabilized predominately by interhelix hydrophobic contacts contributed by the side chains of residues Ala3, Pro4 and Leu6 at the N-terminal loop; Leu11, Leu14, Val17 and Met18 at helix $\alpha 1$; Tyr22, Pro23, Val24, Leu25, Leu26, Phe29, Val30, Leu37, Leu40, Ala43 and Leu44 at helix $\alpha 2$; Ala49, Ala51, Leu52, Ala56 and Phe59 at helix $\alpha 3$; Met66 and Ala68 at the turn between helices $\alpha 3$ and $\alpha 4$; Leu70, Leu71, Tyr74, Leu78 and Ala82 at helix $\alpha 4$; Leu87 at the turn between helices $\alpha 4$ and $\alpha 5$; Ala90, Pro91, Ile94, Met97, Phe101, Ile103, Val104, Ile106, Leu107 and Phe108 at helix $\alpha 5$; and Tyr115 at the C-terminal loop. Helix $\alpha 1$ sits on top of the bundle to form a cap and provides additional stabilization [Fig. 3(c)]. Aside from directly contributing the hydrophobic side chains to the inter-helix hydrophobic contact, helix $\alpha 1$ helps to stabilize the bundle with the side chains of non-hydrophobic residues, which are highly accessible on the surface. The non-hydrophobic residues on helix $\alpha 1$ shield the hydrophobic regions of helices $\alpha 2$ and $\alpha 5$, which are not covered by helices $\alpha 3$ and $\alpha 4$, and the hydrophobic turn connecting helices $\alpha 3$ and $\alpha 4$ from exposure to solvent.

\subsection{The conserved Hpt fold and structural features in the active site}

The protein family of Hpt proteins and domains could be further divided into enzymatically active and inactive orthologs. The active orthologs have a conserved histidine residue that serves as the phosphorelay active site (Ruszkowski et al., 2013). In HptB, this conserved histidine residue, His57, is located near the middle of helix $\alpha 3$. The imidazole side chain of His57 protrudes from the bundle and is exposed to solvent [Figs. 3(c) and 3(d)]. The surrounding residues are featured so as to maximize the accessibility of His57. The hydrogenbonding linkages between Lys60 and Glu79 prohibit their side chains from shielding the imidazole ring of the active-site histidine. High solvent accessibility of the imidazole ring is achieved through the nearby small side-chain volume, which

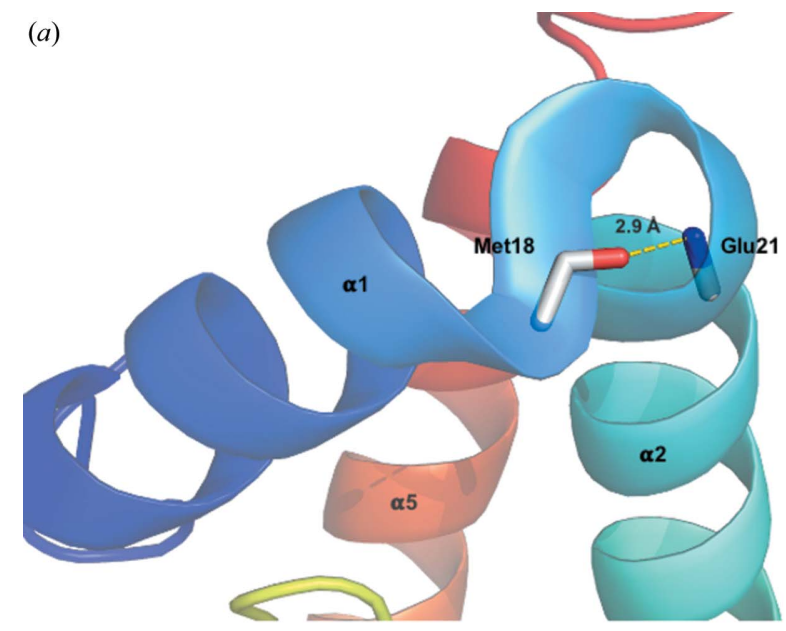

(b)
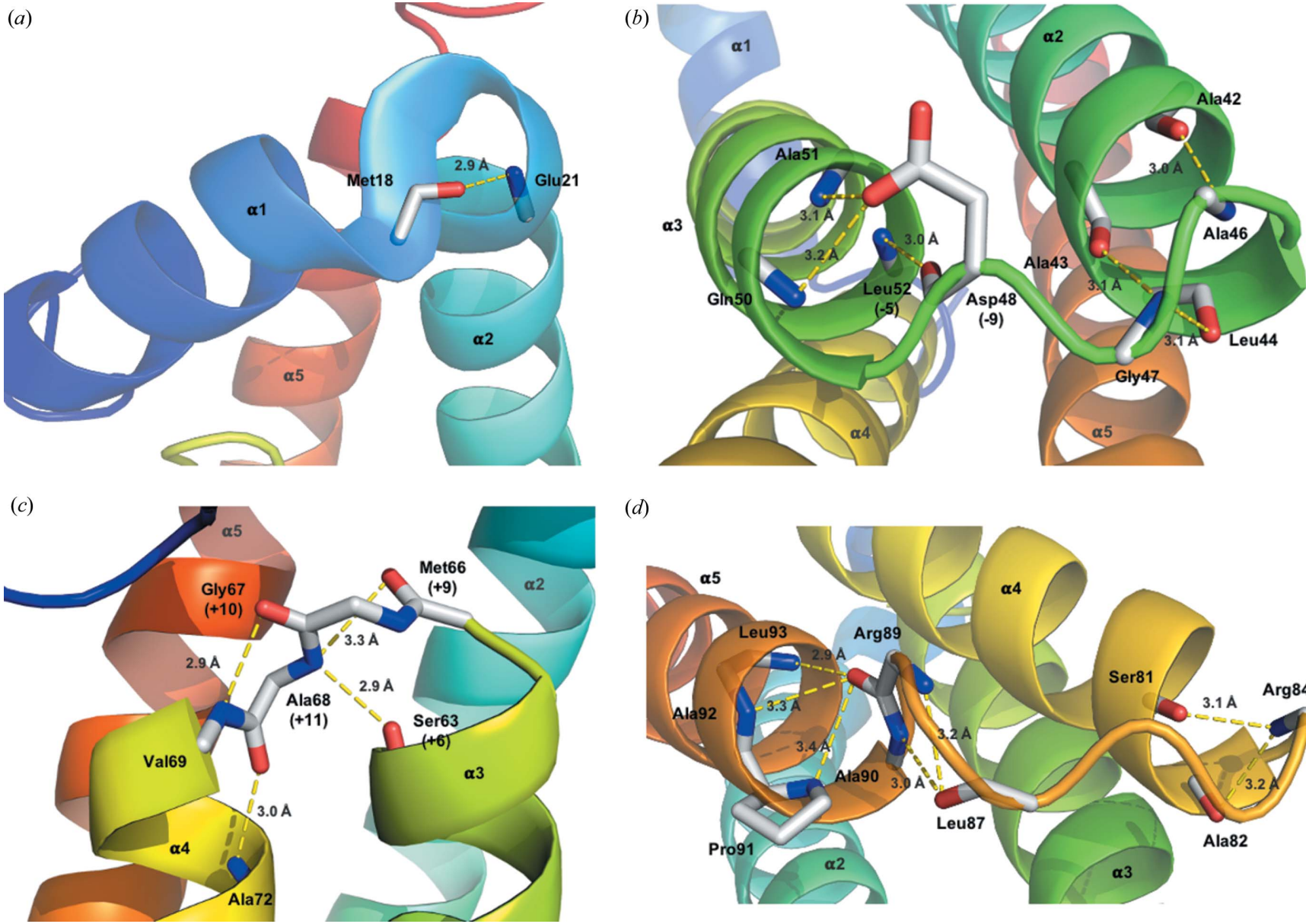

(d)

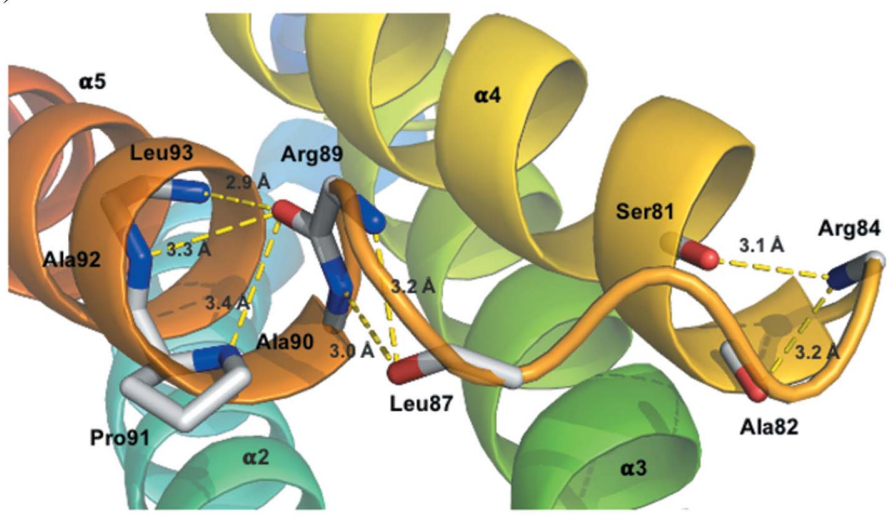

Figure 4

Hydrogen-bond linkages in the inter-helical regions of HptB. The diagrams illustrate the hydrogen bonds (yellow dashed lines) in the inter-helical regions between (a) helices $\alpha 1$ and $\alpha 2$; (b) helices $\alpha 2$ and $\alpha 3$; (c) helices $\alpha 3$ and $\alpha 4$; $(d)$ helices $\alpha 4$ and $\alpha 5$. Carbon, nitrogen and oxygen atoms of the residues involved in the hydrogen-bond linkages are shown as white, blue and red sticks, respectively. Most interactions occur through main-chain atoms of residues. With the exception of Asp48 (at position -9 from the active-site histidine His57) and Pro91, the side chains of residues have been omitted for clarity of the main-chain interactions. The distances listed are the corresponding values averaged over the six molecules in the asymmetric unit. The conserved residues involved in the hydrogen-bond linkages are labeled with numbers in parentheses to indicate their positions from the active-site histidine His57. 


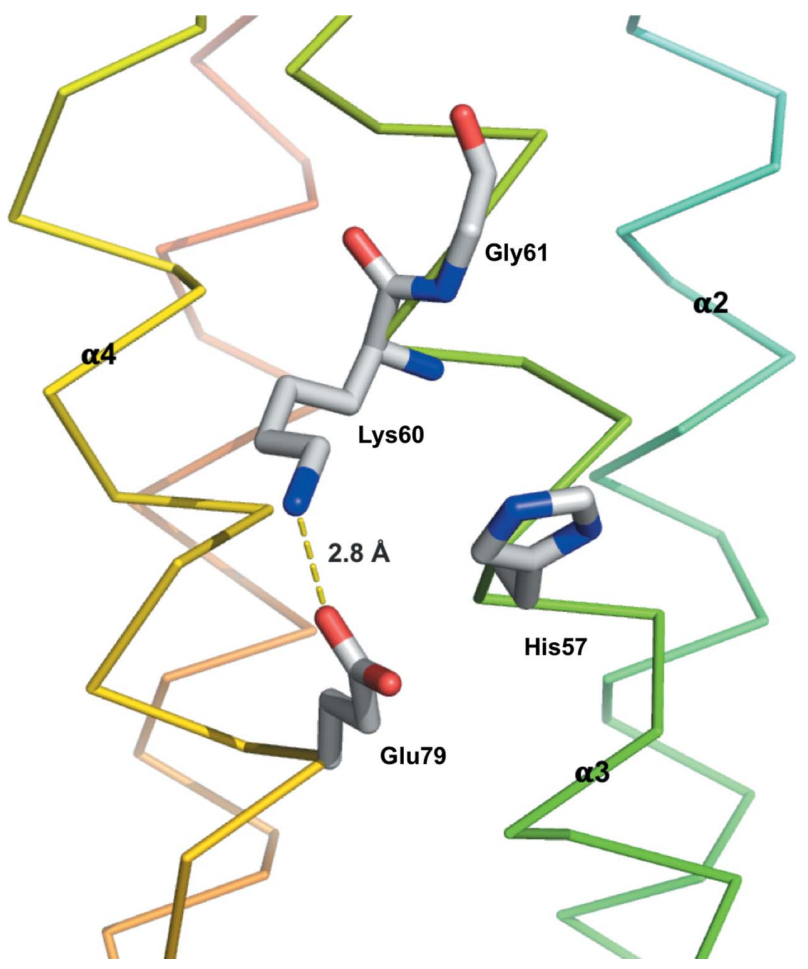

Figure 5

Structural features around the active-site histidine (His57) of HptB. The diagram shows the hydrogen-bond linkage (yellow dashed line) between Lys60 and Glu79 near the active-site histidine (His57) and the spatial position of the highly conserved glycine residue (Gly61) at position +4 from His57. The indicated distance is a value averaged over six molecules in the asymmetric unit. Carbon, nitrogen and oxygen atoms of His57, Lys60, Gly61 and Glu79 are shown as white, blue and red sticks, respectively.

results from the highly conserved glycine residue (Gly61 in $\mathrm{HptB}$ ) at position +4 from the active-site histidine (Fig. 5).

Some conserved residues are related to the stabilization of the structural integrity. Aside from several residues contributed to inter-helix hydrophobic contacts, the residues at positions -5 (Leu52 in HptB), -9 (Asp48), +6 (Ser63), +9 (Met66), +10 (Gly67) and +11 (Ala68) from the active-site histidine are conserved among Hpt proteins/domains with the structures determined listed in Fig. 2(a). The residues at positions -5 and -9 are involved in the hydrogen-bonding linkage between the first helix ( $\alpha 2$ in HptB) and the second helix $(\alpha 3)$, whereas the residues at positions $+9,+10$ and +11 are involved in the hydrogen-bonding linkage between the second helix $(\alpha 3)$ and third helix $(\alpha 4)$ of the bundle in the inter-helical region [Figs. $4(b)$ and $4(c)$ ]. The hydrogenbonding linkages include the hydrogen bonds between (i) the main-chain oxygen of Asp48 at position -9 and the mainchain nitrogen of Leu52 at position -5 [Fig. 4(b)]; (ii) the sidechain oxygen of Asp48 at position -9 and the main-chain nitrogen of Ala51 and Gln50 at positions -6 and -7 , respectively [Fig. 4(b)]; (iii) the main-chain oxygen of Ser63 at position +6 and the main-chain nitrogen of Ala68 at position +11 [Fig. 4(c)]; (iv) the main-chain oxygen of Met66 at position +9 and the main-chain nitrogen of Ala68 at position +11 [Fig. $4(c)$ ]; (v) the main-chain oxygen of Gly67 at position +10 and the main-chain nitrogen of Val69 at position +12 [Fig. 4(c)]; (vi) the main-chain oxygen of Ala68 at position +11 and the main-chain nitrogen of Ala72 at position +15 [Fig. 4(c)]. The $\mathrm{N}$ and $\mathrm{C}$ termini of the second helix ( $\alpha 3)$, at which the active-site histidine is located, are additionally stabilized due to these hydrogen bonds contributed by the conserved residues.

\subsection{Overall structure of PA1611REC}

The recombinant PA1611REC protein comprises amino acids 507-651 of hybrid sensor histidine kinase PA1611 and contains the $\mathrm{C}$-terminal receiver domain. The molecular mass of recombinant PA1611REC with a $6 \times$ His fusion tag is $\sim 18 \mathrm{kDa}$. The crystals of PA1611REC with $\mathrm{Mg}^{2+}$ belong to trigonal space group $P 3_{1} 21$ with one protein molecule in the asymmetric unit. There are 122 amino-acid residues (a.a. 513634) defined in the final atomic model; the electron-density map is clear enough to trace most parts of the main chains and side chains, except residues 507-512 and 635-651 were not seen, likely because of flexibility. The divalent magnesium ion $\left(\mathrm{Mg}^{2+}\right)$ from the crystallization solution was found to be coordinated in the active-site cleft.

The overall structure of PA1611REC possesses a conventional $(\beta / \alpha)_{5}$ topology as observed in other receiver domains of response regulators despite the fact they share lowsequence identities [Fig. $2(b)$ ]. In this $(\beta / \alpha)_{5}$ motif, the fivestranded parallel $\beta$-sheets, which are $\beta 1$ (Glu516-Val520), $\beta 2$ (Arg540-Val544), $\beta 3$ (Ala561-Asp565), $\beta 4$ (Ile591-Thr595) and $\beta 5$ (Asp613-Ala616), are folded in the central core. The core comprises $\beta 1, \beta 3$ and $\beta 4$ which contain conserved hydrophobic residues. The surrounding five $\alpha$-helices are $\alpha 1$ (Pro524-Ser536), $\alpha 2$ (Gly547-Arg556), $\alpha 3$ (Gly573-Arg581), $\alpha 4$ (Gly601-Gln608) and $\alpha 5$ (Arg621-Trp631), among which two (helices $\alpha 1$ and $\alpha 5$ ) are located on one side, whereas the others (helices $\alpha 2, \alpha 3$ and $\alpha 4$ ) are on the opposite side. The $\beta$ strands and $\alpha$-helices are connected with loops. Based on the assignment of the secondary structure from the DSSP web server, helix $\alpha 4$ is composed of two $3_{10}$ helices (Gly601Glu604 and Cys606-Gln608) and separated at Asn605 (Fig. 6). An inspection of the secondary structural geometry shows that the $\varphi$ and $\psi$ angles of Asn605 are -150.3 and $-100.1^{\circ}$, respectively, which deviate from the normal range for a helical structure. Moreover, the adjacent residue Cys606 is positioned structurally closely to Cys566 (on the loop $\beta 3 \rightarrow \alpha 3$ ) to form a disulfide bridge. Interestingly, both Cys606 and Cys566 are unique in P1611REC. Two $33_{10}$ helices separated at Asn605 in helix $\alpha 4$ might be presumably affected by the disulfide formation of Cys606-566. This distinctive structural feature in PA1611REC, which does not exist in other receiver domains of response regulators, might contribute to the interaction with $\mathrm{HptB}$ through the residue on loop $\beta 4 \rightarrow \alpha 4$ discussed later.

\subsection{Metal binding in the active-site cleft of PA1611REC}

In PA1611REC, the conserved aspartate residue, Asp565, responsible for phosphoryl group binding is located in the active-site cleft formed by loops $\beta 1 \rightarrow \alpha 1$ (Glu521 to Asn523), 
(a)

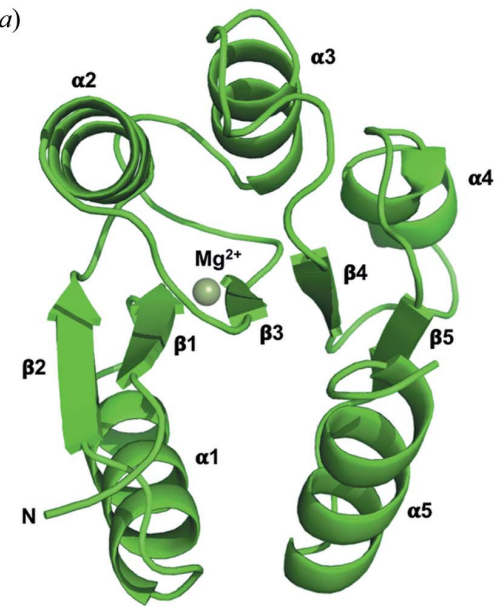

(b)

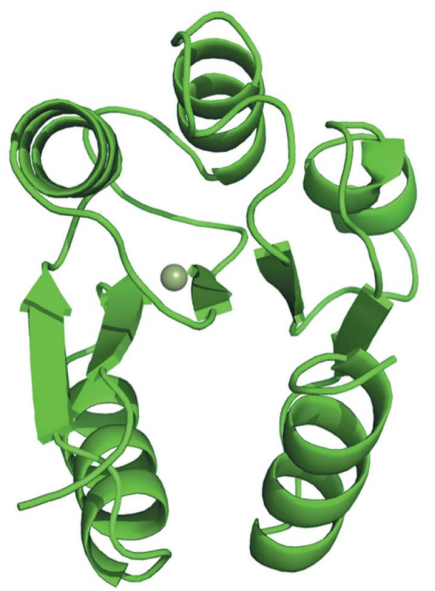

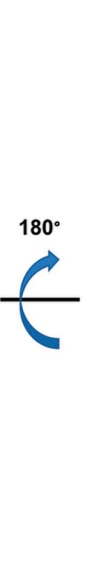
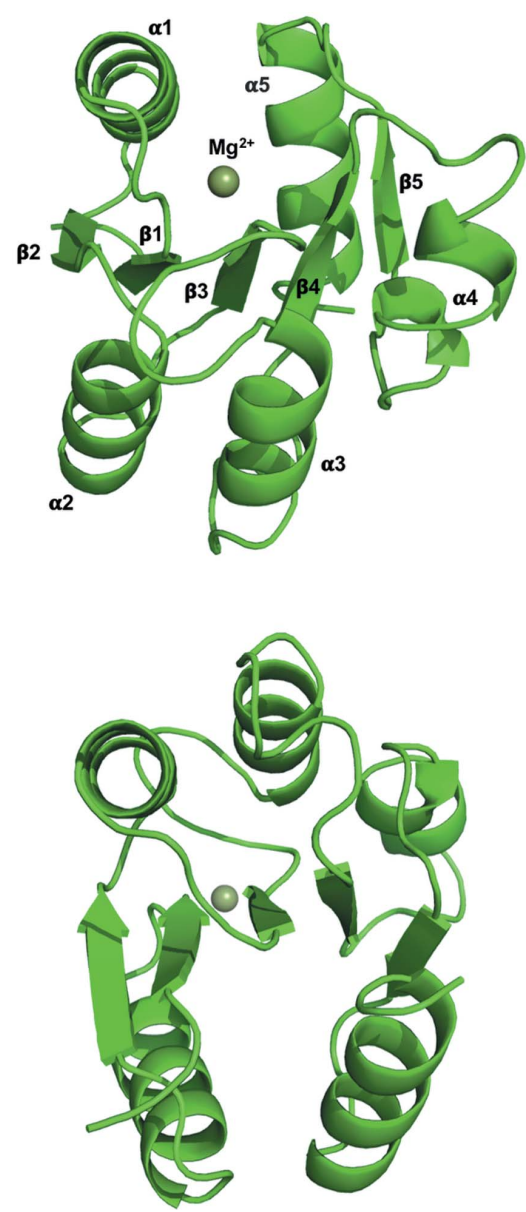

Figure 6

Overall structure of PA1611REC. (a) Ribbon diagram (green) illustrating the secondarystructure elements in PA1611REC. The $\beta$-strands and $\alpha$-helices are numbered sequentially $\beta 1$ to $\beta 5$ and $\alpha 1$ to $\alpha 5$, respectively, from the $\mathrm{N}$ - to the $\mathrm{C}$-terminus. $\mathrm{Mg}^{2+}$ is shown as a light green sphere. (b) Stereoview of the overall structure of PA1611REC with $\mathrm{Mg}^{2+}$ in the active-site cleft (PA1611REC-Mg ${ }^{2+}$ ). $\beta 2 \rightarrow \alpha 2$ (Ala545 to Asp546) and $\beta 3 \rightarrow \alpha 3$ (Cys566 to Asp572) [Fig. 7(a)]. The hydrogen bond between the carbonyl oxygen of Cys566 and the amide nitrogen of Gly573 on helix $\alpha 3$ contributes to the stabilization of the active-site cleft. Previous studies have shown that the binding of divalent metal ions in the active-site cleft is essential to activate the receiver domain (Stock et al., 1993). The surface electrostatic potential around the active-site cleft of PA1611REC is highly negatively charged and suitable to accommodate metal ions [Fig. 7(a)]. The divalent magnesium ion $\left(\mathrm{Mg}^{2+}\right)$ is hexacoordinated, of which three positions coordinate with three water molecules, and three with the main-chain oxygen atom of Arg567 and the side-chain oxygen atoms of Asp522 and Asp565. The three water molecules coordinated to the magnesium ion form further hydrogen bonds with the main-chain oxygen atom of Arg567 and the side-chain oxygen atoms of Asp522 and Asp565. Moreover, one of these water molecules also interacts with Glu521 via hydrogen bonding [Fig. 7(b)].

\subsection{The docking model of the HptB- PA1611REC complex}

The key point in phosphorelay is the recognition between Hpt proteins/ domains and receiver domains of $\mathrm{HK}$ or RR. The complex structures of (a)

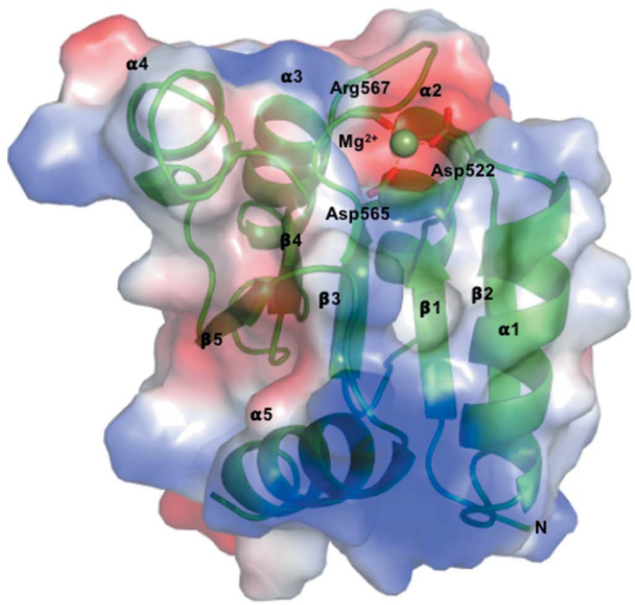

(b)

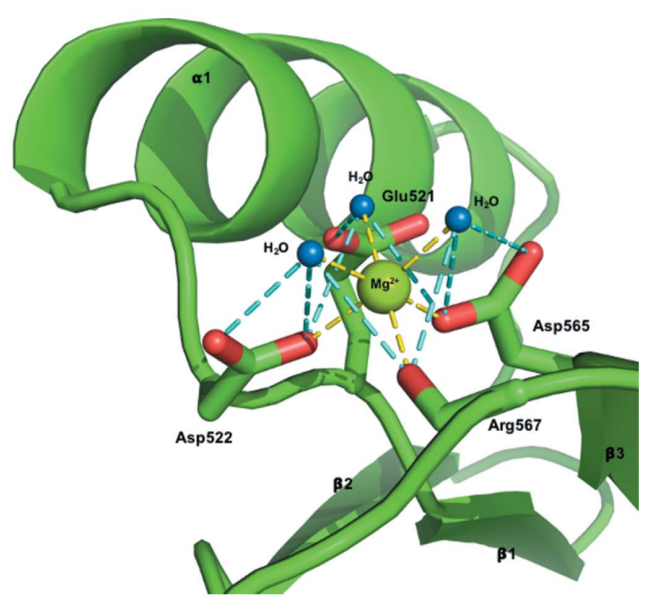

Figure 7

Binding of a divalent metal ion in the active-site cleft of PA1611REC. (a) Diagram of the surface electrostatic potential of PA1611REC. Negatively charged regions are colored red and positively charged regions blue, respectively. Divalent metal ion $\mathrm{Mg}^{2+}$ (shown as a light green sphere) is located in the negatively charged active-site cleft and interacts with Asp522, Asp565 and Arg567 (shown as sticks). (b) Spatial position of the residues and water molecules involved in the interactions with $\mathrm{Mg}^{2+}$. The six coordination positions of $\mathrm{Mg}^{2+}$ are indicated with yellow dashed lines, whereas the interactions between the residues and water molecules are indicated with cyan dashed lines. Oxygen atoms of Glu521, Asp522, Arg567 and Asp565 are shown as red sticks; water molecules are displayed as small blue spheres. 
SLN1-R1/YPD1 (PDB entry 2r25; Zhao et al., 2008) and AHK5 RD $/$ AHP1 (PDB entry 4euk) have highlighted that there is a general shape complementarity between the kidneyshaped Hpt protein/domain and the convex surface of the receiver domain (Zhao et al., 2008; Bauer et al., 2013). The transfer of the phosphoryl group between PA1611 and HptB was confirmed with an in vitro phosphorelay assay using $[\gamma-$ 32P]ATP in our previous work (Lin et al., 2006; Hsu et al., 2008); however, our attempts to obtain the crystal structure of HptB in complex with the receiver domain of PA1611 were unsuccessful. We thus modeled the HptB-PA1611REC complex with the HADDOCK web server to elucidate the interactions between $\mathrm{HptB}$ and the cognate receiver domain.

(a)
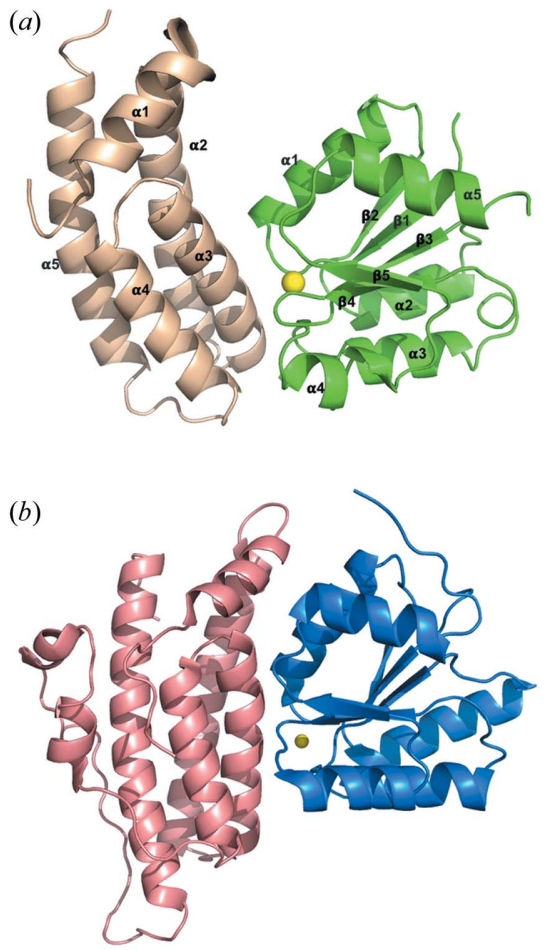

(c)

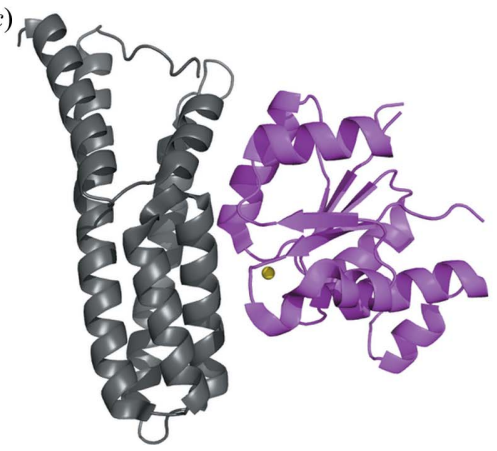

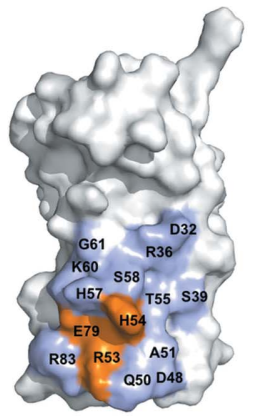
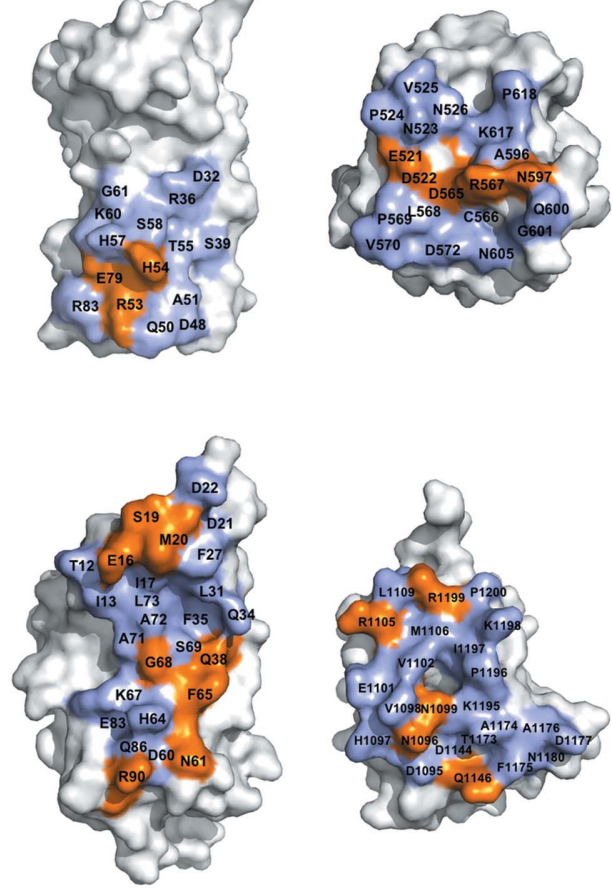

Figure 8

Overall structure and interface of (a) the HptB-PA1611REC complex model and the complexes of

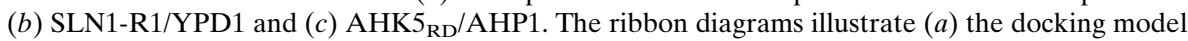
of the HptB-PA1611REC complex and the structures determined of (b) SLN1-R1/YPD1 and (c) AHK5 $5_{\mathrm{RD}} / \mathrm{AHP} 1$ complexes. The molecules of $\mathrm{HptB}$, PA1611REC-Mg ${ }^{2+}$, SLN1-R1, YPD1, $\mathrm{AHK} 5_{\mathrm{RD}}$ and AHP1 are colored wheat, green, blue, salmon, magenta and dark gray, respectively. The $\mathrm{Mg}^{2+}$ ion is shown as a yellow sphere. The interface of the complexes, analyzed using the PDBePISA web server, is shown in the surface presentation. The residues involved in the formation of hydrogen bonds or salt bridges are colored orange, whereas the residues contributing to the hydrophobic and van der Waals interactions are colored purple.
Previous studies have shown that the divalent metal ion is involved in the phosphotransfer reactions of the TCS transduction pathway; the relevant ion in vivo is presumably $\mathrm{Mg}^{2+}$ (Stock et al., 2000). Divalent $\mathrm{Mg}^{2+}$ in the structure of PA1611REC was hence taken into consideration in the docking process. Moreover, as mentioned earlier, the structures of six HptBs in one asymmetric unit are essentially the same; thus, we selected one representative protomer for docking with PA1611REC.

According to the docking model, HptB and PA1611 REC dorm a complex in a 1:1 ratio. Most of the interaction 作 helix bundle of $\mathrm{HptB}$, the $\mathrm{N}$-terminus of helix $\alpha 1$ of PA1611REC, and the loops $(\beta 1 \rightarrow \alpha 1$, $\beta 3 \rightarrow \alpha 3$ and $\beta 4 \rightarrow \alpha 4)$ around the activesite cleft of PA1611REC [Fig. 8(a)]. According to an analysis calculated with the PDBePISA web server, there are 14 hydrogen bonds and salt bridges at the interface, in which 15 residues in $\mathrm{HptB}$ and 20 residues in PA1611REC are involved (Fig. 9, Table 3). Among the above 20 residues, the active-site residue Asp565 of PA1611REC also participates in the hydrogen-bonding network between HptB and PA1611REC. Although the active-site residue His57 of $\mathrm{HptB}$ is in the interface of the complex model, it is, however, not involved in the hydrogen bonds or salt bridges between the two proteins [Figs. $8(a)$ and 9]. There are few structural differences between the proteins of the apo form and the HptB-PA1611REC complex model, with an RMSD of $0.44 \AA$ for $\mathrm{HptB}$ and $0.43 \AA$ for PA1611REC. Furthermore, an inspection of of the residues of $\mathrm{HptB}$ involved in the interface of the complex model revealed that their side chains are positioned in consistent and similar orientations among the six HptB protomers in one asymmetric unit, suggesting that the docking model serves the representative complex.

\section{Discussion}

\subsection{Comparison of HptB with other Hpt proteins/domains}

HptB exhibits a low level of overall sequence similarity with other $\mathrm{Hpt}$ proteins/domains [Fig. 2(a)], but they all share a common structural feature as an elongated four-helix bundle, which is kidney-shaped with up-and-down 


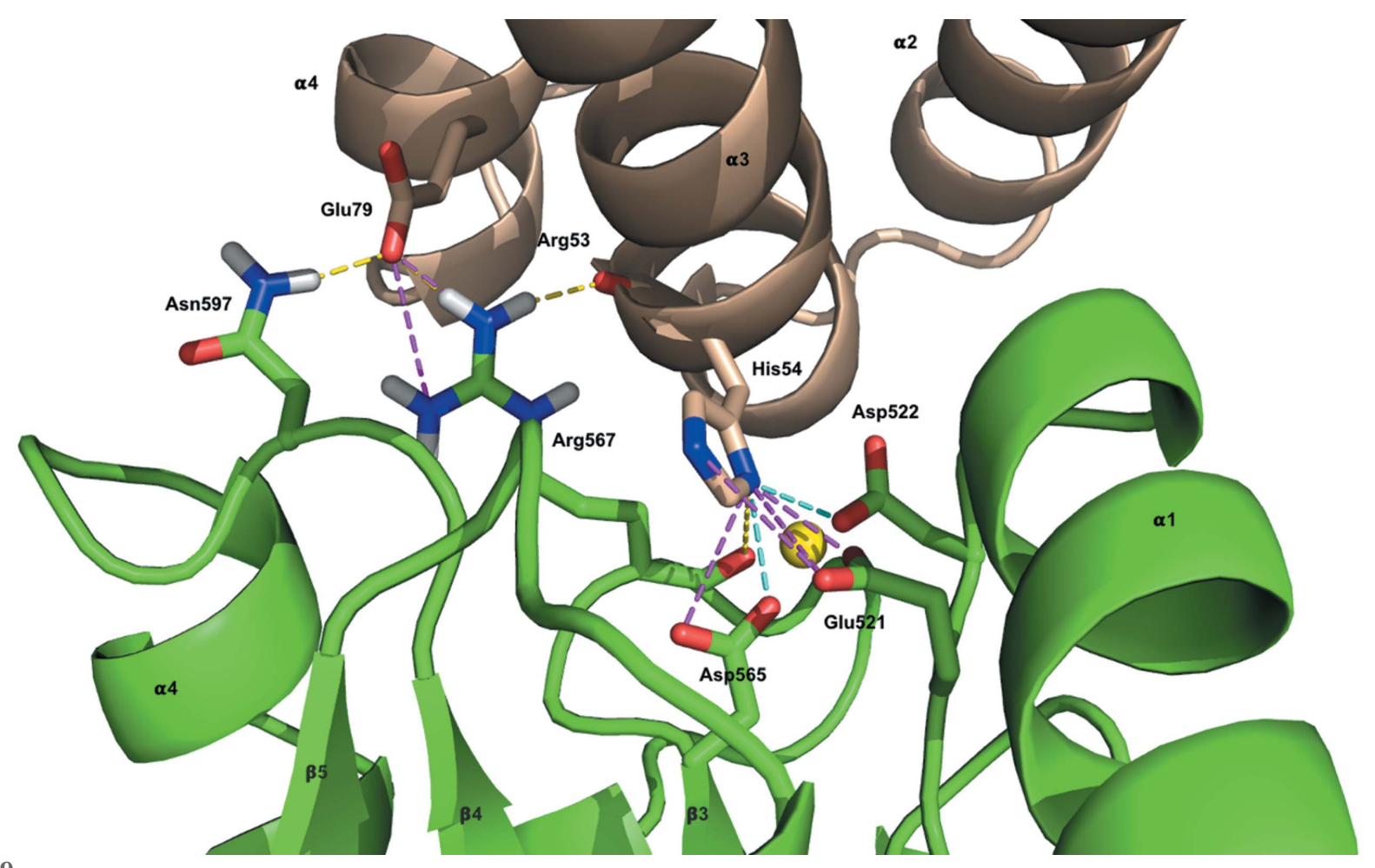

Figure 9

Interactions at the interface of the HptB-PA1611REC complex model. The molecules of HptB and PA1611REC-Mg ${ }^{2+}$ are colored wheat and green, respectively. The $\mathrm{Mg}^{2+}$ ion is shown as a yellow sphere. The main chains and side chains of the residues involved in the interface of the complex model analyzed with PDBePISA are depicted as sticks. Hydrogen bonds and salt-bridge interactions are represented as yellow dashed lines and magenta dashed lines, respectively. The interaction with both hydrogen bonds and salt-bridges are indicated with cyan dashed lines. Nitrogen and oxygen atoms are depicted as blue and red sticks, respectively, and carbon atoms are the same color as the protein molecule.

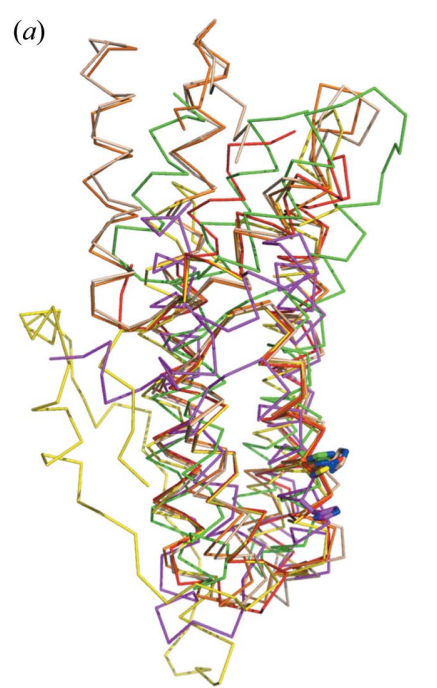

(b)

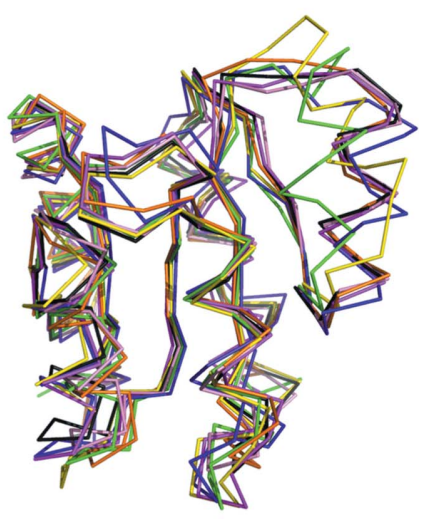

Figure 10

Structure alignment of (a) Hpt proteins/domains and (b) receiver domains listed in Fig. 2. (a) Overall structure of HptB, ZmHP2 and Hpt domain of ArcB, MtHPt1, YPD1 and AHP1 colored red, green, magenta, orange, yellow and wheat, respectively. The common structural motif, the four-helix bundle, is superimposed among these Hpt proteins/ domains. Nitrogen atoms of the active-site histidine are shown as blue sticks; the carbon atoms of the residues are shown as sticks in the same color as the protein. (b) Overall structure of PA1611REC, RR468,

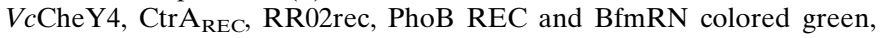
magenta, purple blue, orange, dark gray, yellow and pink, respectively. Among these receiver domains, the main $(\beta / \alpha)_{5}$ architecture is well superimposed, except helix $\alpha 4$ which is is variable, especially helix $\alpha 4$ of PA1611REC. topology and stabilized by inter-helix hydrophobic contacts and hydrogen-bond linkages in the inter-helical regions. The conserved residues are located mainly at the helices forming the four-helix bundle and surrounding the active-site histidine (His57 in $\mathrm{HptB}$ ), indicating that they contribute to the stability of the bundle and maximize the accessibility of the active-site histidine [Fig. 2(a)]. The active-site histidine is located at a similar position, which is near the middle of the second helix ( $\alpha 3$ in $\mathrm{HptB}$ ) of the helix bundle. The major difference is located at the $\mathrm{N}$-terminal helix $(\alpha 1$ in $\mathrm{HptB})$ and $\mathrm{C}$-terminus beyond the four-helix bundle [Fig. 10(a)]. The differences in the segments outside the four-helix bundle might influence the specific interactions between separate Hpt proteins/domains in each species and the cognate receiver domains.

A search for structural homologs with the DALI server (Holm, 2019) showed that HptB bears structural similarity not only to other $\mathrm{Hpt}$ proteins/domains but also to proteins without functional similarities, such as flagellin (PDB entry 5maw; Altegoer, et al., 2018) with a $Z$-score of 9.7, mannose-6phosphate receptor binding protein 1 (PDB entry 1szi; Hickenbottom et al., 2004) with a $Z$-score of 8.7 and cytochrome c' (PDB entry 5b3i; Fujii et al., 2017) with a $Z$-score of 8.5. The four-helix bundle motif is evidently suitable for diverse biological functions (Xu \& West, 1999).

The orientation of helix $\alpha 1$ in HptB is similar to that of helix $\alpha 2$ in AHP1 of Arabidopsis. Helix $\alpha 2$ of AHP1 contributes additional hydrogen bonds and hydrophobic interactions to 
Table 3

PDBePISA analysis at the interface of the HptB-PA1611REC complex model.

\begin{tabular}{|c|c|c|}
\hline HptB residue (atom) & & PA1611REC residue (atom) \\
\hline \multicolumn{3}{|l|}{ Hydrogen bonds } \\
\hline His54 (ND1) & $\leftrightarrow$ & Asp565 (OD2) \\
\hline His54 (ND1) & $\leftrightarrow$ & Asp522 (OD1) \\
\hline His54 (ND1) & $\leftrightarrow$ & Arg567 (O) \\
\hline $\operatorname{Arg} 53(\mathrm{O})$ & $\leftrightarrow$ & Arg567 (HH21) \\
\hline Glu79 (OE1) & $\leftrightarrow$ & Asn597 (HD21) \\
\hline Glu79 (OE1) & $\leftrightarrow$ & Arg567 (HH22) \\
\hline \multicolumn{3}{|l|}{ Salt-bridge interactions } \\
\hline His54 (ND1) & $\leftrightarrow$ & Glu521 (OE1) \\
\hline His54 (ND1) & $\leftrightarrow$ & Glu521 (OE2) \\
\hline His54 (ND1) & $\leftrightarrow$ & Asp565 (OD1) \\
\hline His54 (ND1) & $\leftrightarrow$ & Asp565 (OD2) \\
\hline His54 (ND1) & $\leftrightarrow$ & Asp522 (OD1) \\
\hline His54 (NE2) & $\leftrightarrow$ & Glu521 (OE1) \\
\hline Glu79 (OE1) & $\leftrightarrow$ & Arg567 (NH1) \\
\hline Glu79 (OE1) & $\leftrightarrow$ & Arg567 (NH2) \\
\hline \multicolumn{3}{|c|}{ Hydrophobic and van der Waals interactions } \\
\hline Asp32 & $\leftrightarrow$ & Val525 \\
\hline Arg36 & $\leftrightarrow$ & Asn523 \\
\hline Arg36 & $\leftrightarrow$ & Pro524 \\
\hline Arg36 & $\leftrightarrow$ & Val525 \\
\hline Ser39 & $\leftrightarrow$ & Pro524 \\
\hline Asp48 & $\leftrightarrow$ & Pr0569 \\
\hline Asp48 & $\leftrightarrow$ & Val570 \\
\hline Gln50 & $\leftrightarrow$ & Cys566 \\
\hline Gln50 & $\leftrightarrow$ & Leu568 \\
\hline Gln50 & $\leftrightarrow$ & Pro569 \\
\hline Gln50 & $\leftrightarrow$ & Asp572 \\
\hline Gln50 & $\leftrightarrow$ & Asn605 \\
\hline Ala51 & $\leftrightarrow$ & Pro569 \\
\hline Thr55 & $\leftrightarrow$ & Asn523 \\
\hline His57 & $\leftrightarrow$ & Ala596 \\
\hline His57 & $\leftrightarrow$ & Lys617 \\
\hline His57 & $\leftrightarrow$ & Pro618 \\
\hline Ser58 & $\leftrightarrow$ & Asn523 \\
\hline Ser58 & $\leftrightarrow$ & Asn526 \\
\hline Lys60 & $\leftrightarrow$ & Lys617 \\
\hline Gly61 & $\leftrightarrow$ & Pro618 \\
\hline Arg83 & $\leftrightarrow$ & Gln600 \\
\hline Arg83 & $\leftrightarrow$ & Gly601 \\
\hline Arg83 & $\leftrightarrow$ & Asn605 \\
\hline
\end{tabular}

the interface with the receiver domain of AHK5 (AHK5 $\left.5_{\mathrm{RD}}\right)$. Compared with the AHK $5_{\mathrm{RD}}-\mathrm{AHP} 1$ complex, the $\mathrm{Che} \mathrm{A}_{3} \mathrm{HP} 1$ of $R$. sphaeroides, which lacks extra helices outside the conserved Hpt bundle, has shown a smaller contact area and affinity with its partner, $\mathrm{CheY}_{6}$. The difference in affinity indicates that the extra helix at the N-terminus of the fourhelix bundle might play an important role in the interaction with the cognate receiver domains (Bauer et al., 2013). Our docking model showed, however, that helix $\alpha 1$ of HptB is not involved in the interaction with the receiver domain of PA1611. The major role of this helix might therefore be to stabilize the four-helix bundle of $\mathrm{HptB}$ or to interact with other subjects, such as another domain of PA1611 or other proteins.

4.2. Structural features of $\mathrm{HptB}$ facilitates its interaction with receiver domains

The hydrogen-bond linkages around the side chains of the residues near the active-site histidine have been suggested to facilitate its accessibility to the phosphoryl group and to increase the nucleophilicity of the nitrogen atom at the imidazole ring to improve the efficiency of phosphotransfer (Xu \& West, 1999). Moreover, the glycine residue (Gly61 in $\mathrm{HptB}$ ) at position +4 from the active-site histidine (His57 in $\mathrm{HptB}$ ) is located on its adjacent ridge in the second helix of the bundle ( $\alpha 3$ in $\mathrm{HptB}$ ) and seems to carve a space for the histidine to expose its imidazole side chain to solvent. It has been suggested that, if an amino acid with a larger side chain replaces glycine, the activity of the active-site histidine to accept and to transfer a phosphoryl group would be disturbed (Kato et al., 1997). In YPD1 and the Hpt domain of ArcB, the phosphorylation efficiencies were decreased when this glycine was replaced by Gln or Asp (Sugawara et al., 2005). Aside from the active-site histidine and the glycine residue at position +4 , the lysine residue at position +3 (Lys60 in HptB) is conserved throughout all active Hpt orthologs. The conservation and position imply that this lysine residue is involved in the phosphorelay process. The phosphorylation efficiency of a mutant in which the +3 lysine was substituted with alanine was decreased in YPD1 (Janiak-Spens \& West, 2000). In HptB, Lys60 contributes to the formation of the positively charged region around the active-site histidine, which may help to neutralize the negatively charged phosphoryl group as well as form hydrogen bonds to the oxygen atoms of phosphorylated histidine, providing an additional stabilizing force.

Although the sequence identity is low among Hpt proteins/ domains, all Hpt proteins/domains share a common structural feature and active site, with some conserved residues that could be important for the structure and function described above. The key structural feature for the interactions between $\mathrm{Hpt}$ proteins/domains and receiver domains has been proposed to be the general shape complementarity between them, observed in several TCSs (Kato et al., 1997; Zhao et al., 2008; Bauer et al., 2013). In contrast, non-conserved residues might be important for the specific recognition of molecular partners (Xu \& West, 1999; Xu et al., 2009). Although some Hpt proteins/domains could interact with non-cognate partners in vitro and in vivo, the rates of phosphotransfer in these cases might be substantially less than with cognate partners (Rogov et al., 2004; Xu et al., 2009). Because a different arrangement of amino acid residues would alter the affinity and specificity of the interaction between each $\mathrm{Hpt}$ and receiver domain, one reasonably suspects that the presence of Hpt proteins/domains could help to diminish or prevent the cross-interaction between distinct pathways in a TCS (Sugawara et al., 2005; Xu et al., 2009).

Aside from the general shape matching, other structural features might be involved in the interaction between $\mathrm{Hpt}$ proteins/domains and receiver domains. For example, some Hpt proteins/domains have a hydrophobic cavity in the proximity of the active-site histidine. The hydrophobic interaction has been proposed to contribute to the formation of the Hpt-REC complex; mutation of the hydrophobic residues in that cavity would decrease the phosphotransfer activity (Kato et al., 1999a; Rogov et al., 2004). The active-site histidine (His57) of HptB is surrounded largely by positively charged 
residues. We hence suggest that the electrostatic interactions and hydrogen bonding also help the binding of $\mathrm{HptB}$ and its partner in vivo. Based on an analysis of the complex model with the PDBePISA web server, the residues involved in the interface between HptB and PA1611REC contribute to the hydrogen bonds and salt bridges as well as hydrophobic and van der Waals interactions [Figs. 8(a) and 9, Table 3].

\subsection{Comparison of PA1611REC with other receiver domains}

The receiver domains typically exist in response regulators and also in some sensor histidine kinases in the hybrid TCS. Although the sequence similarity is not high, the receiver domain of PA1611 shares structural and functional homology with most bacterial receiver domains [Figs. $2(b)$ and $10(b)$ ] (Lin et al., 2006; Hsu et al., 2008). The structure alignment of the receiver domains listed in Fig. 2(b) shows that the secondary structures are organized in a similar pattern with a typical $(\beta / \alpha)_{5}$ topology [Fig. 10(b)]. The central $\beta$ strands and the regions around the active-site cleft are highly conserved [Fig. 2(b)]. In contrast, the region containing helix $\alpha 4$ and the loop $\beta 4 \rightarrow \alpha 4$ displays a large variability, which causes an overall RMSD above $0.6 \AA$ between PA1611REC and other receiver domains listed in Fig. 2(b). The RMSD values between PA1611REC- $\mathrm{Mg}^{2+}$ and RR468, $V c$ CheY4, CtrA ${ }_{\mathrm{REC}}$, RR02rec, PhoB REC and BfmRN are 0.92, 0.95, 0.67, 0.79, 0.78 and $0.98 \AA$, respectively.

The conserved residues of the receiver domains are located mainly on $\beta$-strands and $\alpha$-helices. Aside from the aspartate residue that is directly phosphorylated (Asp565 in PA1611REC), highly conserved residues are involved in the process of phosphorelay. The aspartate residue at the end of $\beta 1$ (Asp522 in PA1611REC) participates in the binding of the divalent metal ion. The structure of the $\mathrm{CheY}$ receiver domain with beryllofluoride (phosphoryl group analog) in the activesite cleft (PDB entry 1fqw; Lee et al., 2001) shows that the ligand is located close to the metal ion and is stabilized by $\mathrm{Mg}^{2+}$, conserved Thr87 on $\beta 4$ with a hydrogen bond, and conserved Lys109 on $\beta 5$ by salt-bridge interactions (Lee et al., 2001). We hence suggest that Thr595 (Thr87 in CheY) and Lys617 (Lys109 in CheY) in PA1611REC would also interact with the phosphoryl group and stabilize the conformation of the activated receiver domain during phosphorylation. The alanine residue (Ala596 in PA1611REC) following Thr595 was thought to improve access of the conserved $\mathrm{Thr} / \mathrm{Ser}$ to the phosphorylation site (Bourret, 2010).

Although the $\alpha \rightarrow \beta$ loops opposite the active-site cleft demonstrate greater variability in sequence and length, the $\beta 3 \rightarrow \alpha 3$ loop near the active-site cleft is highly conserved. This loop has been suggested to form a universal recognition element across the receiver domain superfamily (Usher et al., 1998). Moreover, an inspection of temperature $B$-factors of PA1611REC reveals that the $\beta 3 \rightarrow \alpha 3$ loop and the other two loops $(\beta 1 \rightarrow \alpha 1, \beta 2 \rightarrow \alpha 2)$ surrounding the active-site cleft are relatively rigid, compared with the loops away from the active site (see Fig. S1 of the supporting information). In our complex model, this $\beta 3 \rightarrow \alpha 3$ loop participates in the interac- tion with $\mathrm{HptB}$. We hence suggest that the $\beta 3 \rightarrow \alpha 3$ loop in PA1611REC would be involved in the binding with HptB in vivo.

\subsection{Structural alterations in receiver domains}

Conformational changes due to phosphorylation have been assumed to be common in proteins related to signal transduction. For example, in E. coli CheY, a series of structural changes occur near the active-site cleft during phosphorylation (Cho et al., 2000; Lee et al., 2001). The structures of Thermotoga maritima CheY (TMY) indicated that Ser82 and Phe101 might be sensitive to the phosphorylation status of the active-site aspartate and alter the conformation simultaneously. In the unphosphorylated state, the side chain of Phe101 is exposed to solvent; the side-chain hydroxyl of Ser82 points toward Phe101. After TMY is phosphorylated, the sidechain hydroxyl of Ser82 is oriented towards the active site; the aromatic ring of Phe101 is buried (Usher et al., 1998). Similar conformational changes have been reported for the residue substitutions in other receiver domains (Cho et al., 2000; Lee et al., 2001; Zhao et al., 2008). In our determined structure of PA1611REC, there is no phosphate group in the active-site cleft. The side chain of Tyr614 (Phe101 in TMY) is exposed to solvent; the side chain of Thr595 (Ser82 in TMY) points towards it. The results suggest that our PA1611REC is in an inactivated form; there might be conformational changes that occur in the side chain of Thr595 and Tyr614 after phosphorylation, similar to the residue substitutions in E. coli $\mathrm{CheY}$ and TMY.

Helix $\alpha 4$ and its adjacent regions display a great variability in several structures of RR receiver domains. In previous studies, these regions were shown to undergo conformational changes in the activated receiver domain (Cho et al., 2000; Lee et al., 2001; Zhao et al., 2008). Aside from helix $\alpha 4$, the Nterminus of helix $\alpha 1$ has been reported to be partially unfolded when CheY interacts with cognate Hpt proteins/ domains (Kato et al., 1999a). The conformational alterations of activated REC are varied. For example, in the YPD1/SLN1$\mathrm{R} 1$ complex with $\mathrm{Mg}^{2+}$ and $\mathrm{BeF}_{3}{ }^{-}$, conformational changes of a significant number were observed in comparison with the apo complex. In contrast, there are very few conformational changes in the Spo0B/Spo0F- $\mathrm{Mg}^{2+}-\mathrm{BeF}_{3}{ }^{-}$complex compared with the apo complex (Varughese et al., 2006; Zhao et al., 2008). Although the loops and some residues may undergo conformational changes during phosphorylation, there is no dramatic structural rearrangement in the main secondarystructure architecture of receiver domains (Lee et al., 2001). For example, the difference is small between the structures of $\mathrm{BeF}_{3}{ }^{-} \mathrm{BfmRN}$ and the apo form BfmRN, with an RMSD of 0.315 across $227 \mathrm{C} \alpha$ atoms (Draughn et al., 2018). The structural differences between PA1611REC in the apo form and the complex model are also small, with an RMSD of $0.43 \AA$.

4.5. Interactions among the receiver domain, metal ion and Hpt

Previous studies have shown that a divalent metal cation helps to stabilize the phosphorylated active-site aspartate 
residue of the receiver domain. In the study of EL_LovR, a protein with only one receiver domain in Erythrobacter litoralis, its lack of divalent metal ion was suggested to create electrostatic repulsion among the negatively charged residues around the active-site cleft which destabilize the protein (Ocasio et al., 2015). The manners for metal binding are similar in most receiver domains, in which three coordination positions occur with the atoms of amino-acid residues and the others with solvent molecules. The structure of the CheY receiver domain with the phosphoryl group analog, beryllofluoride, in the active-site cleft (PDB entry1fqw; Lee et al., 2001) shows that beryllofluoride is located close to the metal ion; $\mathrm{O}_{\delta}$ on the side chain of the conserved active-site aspartate (Asp57 in CheY) can interact with $\mathrm{Mg}^{2+}$ and the beryllium atom.

The studies of CheY have shown that $\mathrm{Mn}^{2+}$ has the same geometry as $\mathrm{Mg}^{2+}$ in the active-site and can also coordinate to Asp13, Asp57, Asn59 and $\mathrm{BeF}_{3}^{-}$(Lee et al., 2001). The structure of PA1611REC with $\mathrm{Ca}^{2+}$ in the active-site cleft was also determined from a crystal grown in a crystallization solution containing $\mathrm{Ca}^{2+}$ (Fig. S2). The overall structures of PA1611REC-Mg ${ }^{2+}$ and PA1611REC-Ca ${ }^{2+}$ are similar with an RMSD of $0.23 \AA$. These results coincide with suggestions from previous studies that the divalent metal ion would not induce a conformational change of the REC active-site cleft, although it is required for the phosphorylation process (Lee et al., 2001).

The overall arrangement between Hpt proteins/domains and REC is similar in complex structures of SLN1-R1/YPD1, AHK $5_{\mathrm{RD}} / \mathrm{AHP} 1$ and our docking model of HptBPA1611REC. However, compared with SLN1-R1/YPD1 and AHK $5_{\mathrm{RD}} / \mathrm{AHP} 1$ complexes, the slight deflection of the relative positions of HptB and PA1611REC results in fewer residues involved in the interface of our complex model according to an analysis with the PDBePISA web server (Fig. 8). The relative positions of the active-site residues in $\mathrm{HptB}$ (His57) and PA1611REC (Asp565) are also slightly different from those in SLN1-R1/YPD1 and AHK5 RD $/ \mathrm{AHP} 1$ complexes because of the minor deflection. We hence suggest the interactions between the two proteins in the HptB-PA1611REC complex model are weaker than those in SLN1-R1/YPD1 and AHK $5_{\text {RD }} /$ AHP1 complexes. Perhaps for this reason we could not directly observe the interaction between $\mathrm{HptB}$ and PA1611REC in the experiment using size-exclusion chromatography, and hence could not successfully obtain suitable crystals of the HptB-PA1611REC complex. Because the transfer of the phosphoryl group between the cytosolic part of PA1611 (a.a. 202-652) and HptB has been confirmed with [ $\gamma$ 32P]ATP in our previous work (Lin et al., 2006; Hsu et al., 2008), we surmise that another part in addition to REC of PA1611 or some other molecule is necessary to enhance the interaction with $\mathrm{HptB}$.

According to previous studies and an analysis with the PDBePISA web server, the active-site histidine residues of Hpt-related proteins in SLN1-R1/YPD1, AHK5 RD $_{\text {AHP1 }}$ and the HptB-PA1611REC model are involved in the interface of the complex but do not participate in the hydrogen-bond network and salt bridges of the binding surface (Figs. 8 and 9;
Table 3). The conserved lysine residue at position +3 and glycine residue at position +4 from the active-site histidine also contribute to the interaction. These two residues in YPD1 and AHP1 are involved in the hydrogen-bond network or salt bridges of the interface, whereas the two residues (Lys60 and Gly61) in HptB are not involved in the hydrogen bonds and salt bridges between the two proteins in the complex model. Previous studies of the SLN1-R1/YPD1 complex described how the side chain of Gln86 in YPD1, which corresponds to Glu79 in HptB, forms a hydrogen bond with Gln1146 of SLN1R1 (Xu et al., 2003). An analysis of the interface in the HptBPA1611REC complex model with the PDBePISA web server showed that Glu79 of $\mathrm{HptB}$ is also involved in the hydrogenbond networks between the two proteins [Figs. 8(a) and 9; Table 3]. Glu79 of HptB might contribute to the interaction with the cognate receiver domain in vivo.

The aspartate residue (Asp565) responsible for phosphoryl group binding in PA1611REC forms a hydrogen bond with His54 of HptB according to an analysis with the PDBePISA web server. In contrast, the conserved aspartate residue in AHK $5_{\mathrm{RD}}$ is not involved in the interface of the complex. Previous studies of the SLN1-R1/YPD1 complex described that this aspartate residue in SLN1-R1 does not participate in the interface of the complex, although our analysis with the PDBePISA web server shows that it is involved in the interaction between SLN1-R1 and YPD1. The N-terminus of helix $\alpha 1$ and the loop $\beta 1 \rightarrow \alpha 1$, which is one of the loops around the active-site cleft, are highly conserved in structures and sequences among SLN1-R1, AHK5 $5_{\mathrm{RD}}$ and PA1611REC. These two segments contribute largely to the interface of the complex. Loop $\beta 3 \rightarrow \alpha 3$ is structurally conserved and contributes largely to the interaction between $\mathrm{HptB}$ and PA1611REC, whereas in loop $\beta 3 \rightarrow \alpha 3$ of SLN1-R1 and AHK $5_{\mathrm{RD}}$, only one non-conserved residue is involved in the binding surface of the complex. Loops $\beta 4 \rightarrow \alpha 4$ of SLN1-R1, AHK5 $5_{\mathrm{RD}}$ and PA1611REC also majorly contribute to the interface of the three complexes, but these loops display a low level of sequence similarity. According to these analyses, we suggest that the loops around the active-site cleft of REC are not only involved in the interaction with $\mathrm{Hpt}$ proteins/domains but also might influence the specificity of recognition.

The properties and distributions of the amino-acid residues involved in the interactions vary across different complexes. The HptB-PA1611REC complex model, SLN1-R1/YPD1 and AHK $5_{\mathrm{RD}} / \mathrm{AHP1}$ are all well conserved in the structures of their components and the overall organization of the complexes. However, the amino-acid residues contributing to hydrogen bonds, salt bridges and non-polar contacts are distributed differently across the interfaces (Fig. 8) (Zhao et al., 2008; Bauer et al., 2013).

\section{Acknowledgements}

We are indebted to the staff at beamlines TLS 13B1, 15A1 and TPS 05A at the National Synchrotron Radiation Research Center (NSRRC) in Taiwan; Masato Yoshimura and Eiki Yamashita at the BL44XU beamline and Taiwan beamline BL12B2 at SPring-8 in Japan for technical assistance. 


\section{Funding information}

This work was supported in part by the Ministry of Science and Technology (MOST) (grant Nos. 105-2311-B-213-001MY3, 107-2923-B-213-001-MY3 and 108-2311-B-213-001MY3 awarded to CJC).

\section{References}

Altegoer, F., Mukherjee, S., Steinchen, W., Bedrunka, P., Linne, U., Kearns, D. B. \& Bange, K. (2018). Sci. Rep. 8, 11552.

Anjali, Y. B., Yanqi, L., Rakesh, P., Katherine, Y., Xiaoxue, M., Ayush, K. \& Kangmin, D. (2019). Int. J. Mol. 20, 1781-1810.

Balasubramanian, D., Schneper, L., Kumari, H. \& Mathee, K. (2013). Nucleic Acids Res. 41, 1-20.

Bauer, J., Reiss, K., Veerabagu, M., Heunemann, M., Harter, K. \& Stehle, T. (2013). Mol. Plant. 6, 959-970.

Bell, C. H., Porter, S. L., Strawson, A., Stuart, D. I. \& Armitage, J. P. (2010). PLoS Biol. 8, e1000306.

Bent, C. J., Isaacs, N. W., Mitchell, T. J. \& Riboldi-Tunnicliffe, A. (2004). J. Bacteriol. 186, 2872-2879.

Biswas, M., Dey, S., Khamrui, S., Sen, U. \& Dasgupta, J. (2013). PLoS One, 8, e73923-e73923.

Bourret, R. B. (2010). Curr. Opin. Microbiol. 13, 142-149.

Capra, E. J., Perchuk, B. S., Lubin, E. A., Ashenberg, O., Skerker, J. M. \& Laub, M. T. (2010). PLoS Genet. 6, e1001220.

Casino, P., Rubio, V. \& Marina, A. (2009). Cell, 139, 325-336.

Cho, H. S., Lee, S. Y., Yan, D., Pan, X., Parkinson, J. S., Kustu, S., Wemmer, D. E. \& Pelton, J. G. (2000). J. Mol. Biol. 297, 543-551.

Draughn, G. L., Milton, M. E., Feldmann, E. A., Bobay, B. G., Roth, B. M., Olson, A. L., Thompson, R. J., Actis, L. A., Davies, C. \& Cavanagh, J. (2018). J. Mol. Biol. 430, 806-821.

Emsley, P., Lohkamp, B., Scott, W. G. \& Cowtan, K. (2010). Acta Cryst. D66, 486-501.

Fujii, S., Oki, H., Kawahara, K., Yamane, D., Yamanaka, M., Maruno, T., Kobayashi, Y., Masanari, M., Wakai, S., Nishihara, H., Ohkubo, T. \& Sambongi, Y. (2017). Protein Sci. 26, 737-748.

Hickenbottom, S. J., Kimmel, A. R., Londos, C. \& Hurley, J. H. (2004). Structure, 12, 1199-1207.

Holm, L. (2019). Bioinformatics, 35, 5326-5327.

Hsu, J. L., Chen, H. C., Peng, H. L. \& Chang, H. Y. (2008). J. Biol. Chem. 283, 9933-9944.

Hubbard, S. J. \& Thornton, J. M. (1993). NACCESS. Department of Biochemistry and Molecular Biology, University College, London, UK.

Janiak-Spens, F. \& West, A. H. (2000). Mol. Microbiol. 37, 136-144. Kabsch, W. \& Sander, C. (1983). Biopolymers, 22, 2577-2637.

Kaserer, A. O., Andi, B., Cook, P. F. \& West, A. H. (2010). Methods Enzymol. 471, 59-75.

Kato, M., Mizuno, T., Shimizu, T. \& Hakoshima, T. (1997). Cell, 88, 717-723.

Kato, M., Shimizu, T., Mizuno, T. \& Hakoshima, T. (1999a). Acta Cryst. D55, 1257-1263.

Kato, M., Shimizu, T., Mizuno, T. \& Hakoshima, T. (1999b). Acta Cryst. D55, 1842-1849.

Krissinel, E. \& Henrick, K. (2007). J. Mol. Biol. 372, 774-797.

Lee, S. Y., Cho, H. S., Pelton, J. G., Yan, D., Berry, E. A. \& Wemmer, D. E. (2001). J. Biol. Chem. 276, 16425-16431.

Lin, C. T., Huang, Y. J., Chu, P. H., Hsu, J. L., Huang, C. H. \& Peng, H. L. (2006). Res. Microbiol. 157, 169-175.

Lyczak, J. B., Cannon, C. L. \& Pier, G. B. (2002). Clin. Microbiol. Rev. 15, 194-222.

Murshudov, G. N., Skubák, P., Lebedev, A. A., Pannu, N. S., Steiner, R. A., Nicholls, R. A., Winn, M. D., Long, F. \& Vagin, A. A. (2011). Acta Cryst. D67, 355-367.

National Nosocomial Infections Surveillance System (2004). Am. J. Infect. Control, 32, 470-485.
Ocasio, V. J., Corrêa, F. \& Gardner, K. H. (2015). Biochemistry, 54, 1353-1363.

Otwinowski, Z. \& Minor, W. (1997). Methods Enzymol. 276, 307-326.

Posas, F., Wurgler-Murphy, S. M., Maeda, T., Witten, E. A., Thai, T. C. \& Saito, H. (1996). Cell, 86, 865-875.

Rodrigue, A., Quentin, Y., Lazdunski, A., Méjean, V. \& Foglino, M. (2000). Trends Microbiol. 8, 498-504.

Rogov, V. V., Bernhard, F., Löhr, F. \& Dötsch, V. (2004). J. Mol. Biol. 343, 1035-1048.

Ruszkowski, M., Brzezinski, K., Jedrzejczak, R., Dauter, M., Dauter, Z., Sikorski, M. \& Jaskolski, M. (2013). FEBS J. 280, 3709-3720.

Skerker, J. M., Perchuk, B. S., Siryaporn, A., Lubin, E. A., Ashenberg, O., Goulian, M. \& Laub, M. T. (2008). Cell, 133, 1043-1054.

Sola, M., Gomis-Ruth, F. X., Serrano, L., Gonzalez, A. \& Coll, M. (1999). J. Mol. Biol. 285, 675-687.

Stock, A. M., Martinez-Hackert, E., Rasmussen, B. F., West, A. H., Stock, J. B., Ringe, D. \& Petsko, G. A. (1993). Biochemistry, 32, 13375-13380.

Stock, A. M., Mottonen, J. M., Stock, J. B. \& Schutt, C. E. (1989). Nature, 337, 745-749.

Stock, A. M., Robinson, V. L. \& Goudreau, P. N. (2000). Annu. Rev. Biochem. 69, 183-215.

Stover, C. K., Pham, X. Q., Erwin, A. L., Mizoguchi, S. D., Warrener, P., Hickey, M. J., Brinkman, F. S., Hufnagle, W. O., Kowalik, D. J., Lagrou, M., Garber, R. L., Goltry, L., Tolentino, E., WestbrockWadman, S., Yuan, Y., Brody, L. L., Coulter, S. N., Folger, K. R., Kas, A., Larbig, K., Lim, R., Smith, K., Spencer, D., Wong, G. K., Wu, Z., Paulsen, I. T., Reizer, J., Saier, M. H., Hancock, R. E., Lory, S. \& Olson, M. V. (2000). Nature, 406, 959-964.

Sugawara, H., Kawano, Y., Hatakeyama, T., Yamaya, T., Kamiya, N. \& Sakakibara, H. (2005). Protein Sci. 14, 202-208.

Terwilliger, T. C. \& Berendzen, J. (1999). Acta Cryst. D55, 849-861.

Thomason, P. \& Kay, R. (2000). J. Cell Sci. 113, 3141-3150.

Touw, W. G., Baakman, C., Black, J., te Beek, T. A., Krieger, E., Joosten, R. P. \& Vriend, G. (2015). Nucleic Acids Res. 43, D364D368.

Usher, K. C., De La Cruz, A. F., Dahlquist, F. W., James Remington, S., Swanson, R. V. \& Simon, M. I. (1998). Protein Sci. 7, 403-412.

Varughese, K. I., Tsigelny, I. \& Zhao, H. (2006). J. Bacteriol. 188, 4970-4977.

Willett, J. W., Herrou, J., Briegel, A., Rotskoff, G. \& Crosson, S. (2015). Proc. Natl Acad. Sci. USA, 112, E3709-E3718.

Winn, M. D., Ballard, C. C., Cowtan, K. D., Dodson, E. J., Emsley, P., Evans, P. R., Keegan, R. M., Krissinel, E. B., Leslie, A. G. W., McCoy, A., McNicholas, S. J., Murshudov, G. N., Pannu, N. S., Potterton, E. A., Powell, H. R., Read, R. J., Vagin, A. \& Wilson, K. S. (2011). Acta Cryst. D67, 235-242.

Wolanin, P. M., Thomason, P. A. \& Stock, J. B. (2002). Genome Biol. 3, reviews301.

Xu, Q., Carlton, D., Miller, M. D., Elsliger, M. A., Krishna, S. S., Abdubek, P., Astakhova, T., Burra, P., Chiu, H. J., Clayton, T., Deller, M. C., Duan, L., Elias, Y., Feuerhelm, J., Grant, J. C., Grzechnik, A., Grzechnik, S. K., Han, G. W., Jaroszewski, L., Jin, K. K., Klock, H. E., Knuth, M. W., Kozbial, P., Kumar, A., Marciano, D., McMullan, D., Morse, A. T., Nigoghossian, E., Okach, L., Oommachen, S., Paulsen, J., Reyes, R., Rife, C. L., Sefcovic, N., Trame, C., Trout, C. V., van den Bedem, H., Weekes, D., Hodgson, K. O., Wooley, J., Deacon, A. M., Godzik, A., Lesley, S. A. \& Wilson, I. A. (2009). J. Mol. Biol. 390, 686-698.

Xu, Q., Porter, S. W. \& West, A. H. (2003). Structure, 11, 1569-1581. Xu, Q. \& West, A. H. (1999). J. Mol. Biol. 292, 1039-1050.

Zhao, X., Copeland, D. M., Soares, A. S. \& West, A. H. (2008). J. Mol. Biol. 375, 1141-1151.

Zundert, G. C. P. van, Rodrigues, J. P. G. L. M., Trellet, M., Schmitz, C., Kastritis, P. L., Karaca, E., Melquiond, A. S. J., van Dijk, M., de Vries, S. J. \& Bonvin, A. M. J. J. (2016). J. Mol. Biol. 428, 720725 . 